\title{
Noradrenergic Signaling Disengages Feedforward Transmission in the Nucleus Accumbens Shell
}

\author{
Kevin M. Manz, ${ }^{1,2,3}$ Benjamin C. Coleman, ${ }^{7}$ Carrie A. Grueter, ${ }^{3,4}$ Brenda C. Shields, ${ }^{8}$ Michael R. Tadross, ${ }^{8}$ and \\ Brad A. Grueter ${ }^{3,4,5,6,7}$ \\ ${ }^{1}$ Medical Scientist Training Program, Vanderbilt University, Nashville, Tennessee 37232, ${ }^{2}$ Neuroscience Graduate Program, Vanderbilt University, \\ Nashville, Tennessee 37232, ${ }^{3}$ Vanderbilt Brain Institute, Vanderbilt University, Nashville, Tennessee 37232, ${ }^{4}$ Department of Anesthesiology, \\ Vanderbilt University Medical Center, Nashville, Tennessee 37232, ${ }^{5}$ Vanderbilt Center for Addiction Research, Vanderbilt University, Nashville, \\ Tennessee 37232, ${ }^{6}$ Department of Molecular Physiology and Biophysics, Vanderbilt University, Nashville, Tennessee 37232, ${ }^{7}$ Department of \\ Pharmacology, Vanderbilt University, Nashville, Tennessee 37232, and ${ }^{8}$ Department of Biomedical Engineering, Duke University, Durham, North \\ Carolina 27708
}

The nucleus accumbens shell (NAcSh) receives extensive monoaminergic input from multiple midbrain structures. However, little is known how norepinephrine (NE) modulates NAc circuit dynamics. Using a dynamic electrophysiological approach with optogenetics, pharmacology, and drugs acutely restricted by tethering (DART), we explored microcircuit-specific neuromodulatory mechanisms recruited by NE signaling in the NAcSh of parvalbumin (PV)-specific reporter mice. Surprisingly, $\mathrm{NE}$ had little direct effect on modulation of synaptic input at medium spiny projection neurons (MSNs). In contrast, we report that NE transmission selectively modulates glutamatergic synapses onto PV-expressing fast-spiking interneurons (PVINs) by recruiting postsynaptically-localized $\alpha_{2}$-adrenergic receptors (ARs). The synaptic effects of $\alpha_{2}$-AR activity decrease PVIN-dependent feedforward inhibition onto MSNs evoked via optogenetic stimulation of cortical afferents to the NAcSh. These findings provide insight into a new circuit motif in which NE has a privileged line of communication to tune feedforward inhibition in the NAcSh.

Key words: adrenergic receptor; feedforward inhibition; neorepinephrine; nucleus accumbens; parvalbumin interneurons; prefrontal cortex

\section{Significance Statement}

The nucleus accumbens (NAc) directs reward-related motivational output by integrating glutamatergic input with diverse neuromodulatory input from monoamine centers. The present study reveals a synapse-specific regulatory mechanism recruited by norepinephrine (NE) signaling within parvalbumin-expressing interneuron (PV-IN) feedforward inhibitory microcircuits. PV-IN-mediated feedforward inhibition in the NAc is instrumental in coordinating NAc output by synchronizing the activity of medium spiny projection neurons (MSNs). By negatively regulating glutamatergic transmission onto PVINs via $\alpha_{2}$-adrenergic receptors (ARs), NE diminishes feedforward inhibition onto MSNs to promote NAc output. These findings elucidate previously unknown microcircuit mechanisms recruited by the historically overlooked NE system in the NAc.

\section{Introduction}

The nucleus accumbens (NAc) receives extensive monoaminergic input from multiple midbrain structures, including the ventral

\footnotetext{
Received Sep. 15, 2020; revised Feb. 4, 2021; accepted Mar. 9, 2021.

Author contributions: K.M.M., B.C.S., M.R.T., and B.A.G. designed research; K.M.M., B.C.C., and C.A.G. performed research; K.M.M., B.C.C., and C.A.G. analyzed data; K.M.M. wrote the paper.

This work was supported by the National Institute on Drug Abuse Grant R01 DA040630 (to B.A.G.), the BRAIN Initiative Grant RF1-MH117055 (to M.R.T.), and the New Innovator Award DP2-MH119425 (to M.R.T.). K.M.M. is supported by the National Institute of General Studies Grant T32 GM108554.

The authors declare no competing financial interests.

Correspondence should be addressed to Brad A. Grueter at brad.grueter@vumc.org.

https://doi.org/10.1523/JNEUROSCI.2420-20.2021

Copyright $\odot 2021$ the authors
}

tegmental area (VTA) and dorsal raphe nucleus. However, norepinephrine (NE), synthesized primarily in dopamine (DA)- $\beta$ hydroxylase (DBH)-containing cells in the nucleus of the solitary tract (NTS) and locus coeruleus, has garnered only modest attention regarding NAc circuit function (Fallon and Moore, 1978; Allin et al., 1988). Relative to other subcortical regions within the reward network, the NAc is sparsely innervated by $\mathrm{DBH}-(+)$ noradrenergic fibers, with immunoreactivity for $\mathrm{DBH}$ highest in the NAc shell (NAcSh; Berridge et al., 1997; Delfs et al., 1998). Accordingly, $\alpha$ and $\beta$ adrenergic receptors (ARs) and the NE transporter (NET) are expressed highest in the NAcSh along a rostral-caudal gradient (Brog et al., 1993; Kerfoot and Williams, 2011; Mitrano et al., 2012). Despite the presence of functional NEergic afferents to the NAc, mechanisms by which noradrenergic signaling 
influences NAc circuit dynamics remain unknown (McKittrick and Abercrombie, 2007).

Central NE signaling contributes to stress-induced reinstatement of drug-seeking behavior by engaging extrastriatal limbic networks, including the bed nucleus of the stria terminalis (BNST), prefrontal cortex (PFC), and VTA (Mantsch et al., 2016; Harris et al., 2018; Giustino et al., 2019). However, the functional significance of NE in the NAc is unclear. Ex vivo studies in rat suggest that NE modulates glutamatergic transmission onto medium spiny projection neurons (MSNs) via presynaptic $\alpha$-ARs (Nicola and Malenka, 1998; Peng et al., 2018). Both $\alpha_{1}$-AR and $\alpha_{2}$-AR subtypes are expressed in the NAc, with ultrastructural analyses showing that $\alpha$-ARs are expressed at presynaptic and postsynaptic domains in the core and shell (Mitrano et al., 2012; Park et al., 2017). Monoaminergic crosstalk between mesoaccumbens dopamine (DA) and NE signaling has also been described (Sánchez-Soto et al., 2016, 2018), indicating that NE, despite a relatively sparse innervation pattern, may exert diverse neuromodulatory effects on NAc circuit output.

A primary circuit element governing NAc output is feedforward inhibition mediated by fast-spiking parvalbumin (PV)expressing interneurons (PV-INs; Wright et al., 2017; Yu et al., 2017; Manz et al., 2019). PV-INs are instrumental in coordinating D1 and D2 DA receptor-expressing MSN output and are required for experience-dependent behavioral plasticity (Yu et al., 2017; Wang et al., 2018; Pisansky et al., 2019). Recent evidence suggests that glutamatergic synapses onto PV-INs exhibit dynamic computational properties that guide NAc-dependent motivational behavior (Manz et al., 2020b). For example, glutamatergic transmission onto PV-INs is gated by synapse-specific plasticity mechanisms that accelerate psychostimulant-induced associative learning ( $\mathrm{Yu}$ et al., 2017). However, it remains unknown how monoaminergic transmission, such as NE signaling, modulates PV-IN-embedded feedforward inhibitory networks in the NAc.

Here, we interrogate the neuromodulatory actions of NE signaling on feedforward inhibitory microcircuit function leveraging a dynamic electrophysiological approach with pharmacology, input-specific optogenetics, and drugs acutely restricted by tethering (DART). We find that NET-regulated NE release decreases glutamatergic synaptic efficacy onto PV-INs in the NAcSh. This effect is specific to NE, as transporter-regulated DA or 5-HT release does not replicate the phenomena. The actions of NE at PV-IN synapses are postsynaptically mediated via $\alpha_{2}$-AR and accompanied by an inward conductance likely confined to dendritic specializations. In contrast to feedforward synapses onto PV-INs, NE signaling minimally affected local glutamatergic and GABAergic synapses onto MSNs. The precise synaptic modifications elicited by NE are sufficient to promote MSN escape from PFC-evoked feedforward inhibition mediated by PV-INs. These data highlight a privileged synaptic locus within feedforward microcircuits through which NEergic transmission modulates NAcSh circuit output.

\section{Materials and Methods \\ Animals}

Animals were bred and housed at Vanderbilt University Medical Center in accordance with IACUC. Male and female mice 7-12 weeks of age were used for all electrophysiological experiments. Experimental mice were housed in groups of three to five per cage on a 12/12 h light/dark cycle with ad libitum access to standard food and water. Breeding cages were given 5LOD chow to improve litter viability. For all electrophysiological experiments examining PV-INs, Cre-induced STOP ${ }^{f l / f l}$-tdTomato mice (Ai9, Gt(ROSA)26Sor ${ }^{\text {tm9(CAG-tdTomato)Hze })}$ obtained from The
Jackson Laboratory (stock no. 007909) were crossed with PV PV-IRESCre mice (PV ${ }^{\mathrm{Cre}}$, Pvalb ${ }^{\text {tml(cre)Arbr/J }}$, stock no. 012358), generating $\mathrm{PV}^{\mathrm{Cre}}$ tdTomato $^{\mathrm{f} / \mathrm{fl}}\left(\mathrm{PV}^{\mathrm{tdT}}\right)$ mice. Experiments requiring optogenetic access to the PFC were performed in C57BL/6J wild-type (WT) and $\mathrm{PV}^{\mathrm{Cre}}$ mice.

\section{Electrophysiology}

Whole-cell patch-clamp electrophysiological recordings were obtained in acute brain slice preparations from $\mathrm{PV}^{\mathrm{tdT}}$ and $\mathrm{WT}$ mice. Mice were euthanized under isoflurane anesthesia after which parasagittal slices $(250 \mu \mathrm{M})$ containing the medial NAcSh were prepared from whole brain tissue using a Leica Vibratome in oxygenated $\left(95 \% \mathrm{O}_{2} ; 5 \% \mathrm{CO}_{2}\right)$ ice-cold $N$-methyl-D-glucamine (NMDG)-based solution $(2.5 \mathrm{~mm} \mathrm{KCl}, 20 \mathrm{~mm}$ HEPES, $1.2 \mathrm{~mm} \mathrm{NaH}_{2} \mathrm{PO}_{4}$, $25 \mathrm{~mm}$ glucose, 93 mм NMDG, 30 mм $\mathrm{NaHCO}_{3}$, $5.0 \mathrm{~mm}$ sodium ascorbate, $3.0 \mathrm{~mm}$ sodium pyruvate, $10 \mathrm{~mm} \mathrm{MgCl}_{2}$, and $0.5 \mathrm{~mm} \mathrm{CaCl}_{2}-2 \mathrm{H}_{2} \mathrm{O}$ ). Slices were then recovered in NMDG-based recovery solution for $10-15 \mathrm{~min}$ at $30-32^{\circ} \mathrm{C}$ before being transferred to a chamber containing artificial CSF (ACSF; $119 \mathrm{~mm} \mathrm{NaCl}, 2.5 \mathrm{~mm}$ $\mathrm{KCl}, 1.3 \mathrm{~mm} \mathrm{MgCl}_{2}-6 \mathrm{H}_{2} \mathrm{O}, 2.5 \mathrm{~mm} \mathrm{CaCl}{ }_{2}-2 \mathrm{H}_{2} \mathrm{O}, 1.0 \mathrm{~mm} \mathrm{NaH}_{2} \mathrm{PO}_{4}-$ $\mathrm{H}_{2} \mathrm{O}, 26.2 \mathrm{~mm} \mathrm{NaHCO}$, and $11 \mathrm{~mm}$ glucose; 287-295 mOsm). All experiments were performed using a Scientifica Slicescope Pro System with continuously-perfused $28-32^{\circ} \mathrm{C}$ ACSF at $2 \mathrm{ml} / \mathrm{min}$. PV-INs in the NAcSh were visualized using Scientifica PatchVision software and differentiated according to the expression of the red tdTomato fluorophore via 530-nm LED light. Additionally, PV-INs were confirmed according to morphologic (size, shape) and biophysical properties (e.g., capacitance, membrane resistance, and AMPAR decay kinetics) and patched with 3-7 $\mathrm{M} \Omega$ recording pipettes (P1000 Micropipette Puller). Whole-cell recordings were performed in a $\mathrm{K}^{+}$based intracellular solution $\left(135 \mathrm{~mm} \mathrm{~K}^{+}\right.$-gluconate, $5 \mathrm{~mm} \mathrm{NaCl}, 2 \mathrm{~mm}$ $\mathrm{MgCl}_{2}, 10 \mathrm{~mm}$ HEPES, $0.6 \mathrm{~mm}$ EGTA, $3 \mathrm{~mm} \mathrm{Na} \mathrm{NTP}_{2}$, and $0.4 \mathrm{~mm}$ $\mathrm{Na}_{2} \mathrm{GTP}$; 285-292 mOsm). Experiments requiring a depolarized command voltage to isolate GABAergic transmission were performed in a $\mathrm{Cs}^{+}$-based intracellular solution $\left(120 \mathrm{~mm} \mathrm{CsMeSO}_{3}, 15 \mathrm{~mm} \mathrm{CsCl,} 8 \mathrm{~mm}\right.$ $\mathrm{NaCl}, 10$ mм HEPES, 0.2 mм EGTA, 10 mм TEA-Cl, 4.0 mм Mg-ATP, $0.3 \mathrm{~mm}$ Na-GTP, $0.1 \mathrm{~mm}$ spermine, and $5.0 \mathrm{~mm}$ QX 314 bromide). Cellattached recordings in voltage-clamp were performed with 5-7 $\mathrm{M} \Omega$ recording pipettes backfilled with ACSF.

For voltage-clamp recordings of glutamatergic transmission, AMPARmediated electrically-evoked EPSCs were obtained at $-70 \mathrm{mV}$ and isolated with $\mathrm{GABA}_{\mathrm{A}} \mathrm{R}$ antagonist, picrotoxin $(50 \mu \mathrm{M})$. Interleaved electrically-evoked and optically-evoked IPSCs (e/oIPSCs) in MSNs were isolated electrochemically at $0( \pm 5) \mathrm{mV}$ using the $\mathrm{E}_{\mathrm{Cl}}$ and $\mathrm{E}_{\mathrm{AMPAR} / \mathrm{NMDAR}}$ of the $\mathrm{Cs}^{+}$-based internal solution. In experiments examining local synaptic transmission, a bipolar electrode was placed at the corticoaccumbens interface and stimulated at $0.1 \mathrm{~Hz}$. Paired pulse ratios (PPRs) were obtained within-experiment by delivering two $0.3-\mathrm{ms}$ duration pulses with a 50-ms interstimulus interval and calculating the amplitude ratio of the second EPSC to the first EPSC (EPSC $/$ EPSC $_{1}$ ) at the indicated time point. In optogenetic experiments examining input-specific synaptic transmission, $473 \mathrm{~nm}$ Cool LED stimulation was delivered at $0.1 \mathrm{~Hz}$ with a $0.3-$ to $1-\mathrm{ms}$ pulse duration. For current-clamp recordings, cells were permitted 5 min after entering whole-cell configuration to equilibrate to the intracellular dialysate, after which a depolarizing plateau potential was established to maintain cells at approximately $-70 \mathrm{mV}$. To assess intrinsic membrane excitability, action potentials (APs) were elicited following 50-pA current steps increasing from -400 to $550 \mathrm{pA}$ with an 800 -ms step duration every 2 min. Input-output experiments exhibiting pronounced depolarization block were omitted from analysis. Cell-attached recordings of AP fidelity were quantified as the percentage of successful APs elicited following eight stimuli at 50\% stimulus intensity. Because of the limited Cre penetrance in PV-IRES-Cre mice, DART manipulations were performed in Tomato-negative MSNs adjacent to Tomatoexpressing PV-INs in the NAcSh. Membrane resistance $\left(\mathrm{R}_{\mathrm{m}}\right)$ and series resistance $\left(R_{S}\right)$ were monitored continuously during all experiments, with $>15 \%$ change in $\mathrm{R}_{\mathrm{S}}$ resulting in the omission of that experiment. Postsynaptic conductances were quantified according to time-locked changes in holding current ( $\mathrm{I}_{\text {holding }}$ ) and membrane resistance obtained within experiment in voltage-clamp configuration. 
$D A R T$

We leveraged the second-generation DART2.0 system, which offers a wider dosing window than its predecessor (Shields et al., 2017). To select PV-INs as the target of DART manipulation, we injected $500 \mathrm{nl}$ of rAAV $_{2 / 1}$-CAG-DIO-HaloTagDART2.0-2A-dTomato-WPRE bilaterally into the NAc of $\mathrm{PV}^{\mathrm{Cre}}$ mice. Mice were given four to six weeks to allow for viral expression before preparation of acute brain slices. Reagents were validated in HT-expressing $[\mathrm{HT}(+)]$ and HT lacking [HT(-)] neurons by examining pharmacosensitivity to low-dose (300 nM) YM90K ${ }^{\text {DART2.0 }}$ (hereafter, YM90K ${ }^{\text {DART }}$ ). At this dose, ambient YM90K ${ }^{\text {DART }}$ has negligible impact on HT(-) neurons but accumulates within minutes to produce a saturating dose on the surface of $\mathrm{HT}(+)$ neurons. Prior characterization of YM90K ${ }^{\text {DART }}$ indicates that its pharmacophore is an AMPAR-specific antagonist, with no impact on the NMDAR nor significant off-target activity in a screen of 30 brain-enriched receptors (Shields et al., 2017).

\section{Stereotaxic surgery}

Six- to eight-week-old male WT or PV ${ }^{\text {Cre }}$ mice were anesthetized using ketamine $(75 \mathrm{mg} / \mathrm{kg}$, i.p.) and dexdomitidor $(0.5 \mathrm{mg} / \mathrm{kg}$, i.p.). Briefly, craniotomies were performed using a drill, AmScope microscope, and World Precision Instruments Aladdin Al-2000 syringe pump hydraulic system. The following coordinates were used based on The Mouse Brain in Stereotaxic Coordinates: PFC (AP 1.4, ML $\pm 0.5, \mathrm{DV}-2.9 \mathrm{~mm}$ ) and NAc (AP1.20, ML $\pm 0.5, \mathrm{DV}-4.0 \mathrm{~mm}$ ). Injection sites were located using Leica AngleTwo Stereotaxic software. AAV-CaMKII-ChR2-eYFP (Addgene) was injected into the PFC at $100 \mathrm{nl} / \mathrm{min}$. Mice were revived using antisedan (atipamezole, $0.5 \mathrm{mg} / \mathrm{kg}$, i.p.) and treated with ketoprofen $(5 \mathrm{mg} / \mathrm{kg}$, i.p.) for $3 \mathrm{~d}$ postoperatively. ChR2 expression and anatomic specificity were validated empirically according to the expression of eYFP in the medial PFC and the presence of high-fidelity AMPARmediated monosynaptic oEPSCs, as described previously (Turner et al., 2018; Manz et al., 2020a).

\section{Traditional pharmacology}

1-Naphthyl acetyl spermine (NASPM) trihydrochloride, NBQX disodium salt, picrotoxin, $(R)$-tomoxetine $(\mathrm{TMx}) \mathrm{HCl}$, desipramine $\mathrm{HCl}$, GBR12783 dihydrochloride, noradrenaline bitartrate (NE), atipamezole $\mathrm{HCl}$, guanfacine $\mathrm{HCl}$, propranolol, $(R)$-phenylephrine $(\mathrm{PE})$, and phentolamine mesylate were purchased from Tocris Biosciences. $\mathrm{BaCl}_{2}$, cocaine $(\mathrm{COC}) \mathrm{HCl}, \mathrm{GDP}_{\beta} \mathrm{S}$ trilithium salt, and fluoxetine $\mathrm{HCl}$ were purchased from Sigma-Aldrich.

\section{Statistics and data analysis}

Electrophysiological experiments were analyzed using Clampfit 10.4 and GraphPad Prism v7.0. Changes in baseline EPSC amplitude, coefficient of variance $(\mathrm{CV})$, and PPR were calculated by comparing mean values during 5-min intervals specified in each time course to baseline PPR and $\mathrm{CV}$ values. A depression was defined as a significant difference in EPSC, eIPSC, or eIPSC amplitude from baseline calculated during the time interval specified in the recording. Paired or unpaired $t$ tests were used to analyze statistical differences between datasets. Sidak's post hoc analyses were used for analyses requiring multiple comparisons. Power analyses were performed with preliminary data during the acquisition of each new dataset. The sample size obtained from each power analysis calculation was then compared with sample sizes reported in the literature for similar experiments. Errors bars depicted in figures represent SEM. For all analyses, $\alpha$ was set as 0.05 , with $p$ values $<\alpha$ indicating a statistically significant difference.

\section{Results}

\section{Feedforward glutamatergic synapses onto PV-INs are negatively regulated by noradrenergic signaling in the NAcSh}

To distinguish PV-INs from MSNs and other cell-types in the NAcSh, we bred PV ${ }^{\text {Cre }}$ mice with tdTomato ${ }^{\text {fl-STOP-fl }}\left(\mathrm{PV}^{\mathrm{tdT}}\right)$ mice in which the PV promoter drives Cre-dependent tdTomato (tdT) expression (Fig. 1A). Using whole-cell patch-clamp electrophysiology in ex vivo brain slices, we first confirmed that $\operatorname{tdT}(+)$ cells correspond functionally to PV-INs by comparing the biophysical properties of $\operatorname{tdT}(+)$ cells with $\operatorname{tdT}(-)$ cells (putative MSNs). Following somatic current injection, $\operatorname{tdT}(+)$ cells exhibited high-frequency AP firing with short-duration waveforms and steep after-hyperpolarizations (AHPs), consistent with the fast-spiking electrophysiological profile of PV-INs in the NAc (Yu et al., 2017; Manz et al., 2020b). In contrast, tdT(-) cells displayed a regular-spiking interval AP profile with pronounced, low-amplitude AHPs (Fig. 1B). Thus, tdT $(+)$ cells correspond to fast-spiking PV-INs in the NAcSh.

PV-INs require robust glutamatergic input to initiate feedforward inhibition of MSNs in the NAcSh (Yu et al., 2017; Scudder et al., 2018). To assess whether monoamines broadly regulate glutamatergic synaptic strength onto PV-INs, we prepared ex vivo brain slices from $\mathrm{PV}^{\mathrm{tdT}}$ mice and recorded electricallyevoked EPSCs from PV-INs in the NAcSh (Fig. 1C). To discern whether monoamine transporter (MAT)-regulated transmitter release modulates glutamatergic transmission onto PV-INs, we bath-applied selective pharmacological reuptake inhibitors for the DA transporter (DAT), 5-HT transporter (SERT), and NET. Bath-application of selective DAT inhibitor, GBR12873 (1 $\mu \mathrm{M})$, or SERT inhibitor, fluoxetine $(1 \mu \mathrm{M})$, failed to evoke a change in EPSC amplitude (DAT, 95.76 $\pm 3.98 \%, n=7, p=0.144$; SERT, $94.49 \pm 4.59 \%, n=6, p=0.221$; Fig. $1 D-F)$. In contrast to DAT and SERT blockade, desipramine $(10 \mu \mathrm{M})$, a tricyclic NET inhibitor with minimal actions at DAT or SERT, resulted in a robust decrease in EPSC amplitude at PV-IN synapses (NET, $37.17 \pm$ $3.77 \%, n=5, p<0.001$; Fig. $1 D-F)$. These data suggest that NETregulated NE release in the NAcSh regulates glutamatergic synaptic strength onto PV-INs.

\section{Noradrenergic control of glutamatergic transmission occurs at PV-in but not MSN synapses}

To ensure that the effects of desipramine were not because of atypical properties of the drug itself, we repeated experiments with the more selective NET inhibitor, TMx (10 $\mu \mathrm{M}$; Matsui and Alvarez, 2018). TMx recapitulated the effects of desipramine on EPSC amplitude, reaffirming the observation that endogenouslyreleased NE from NET-expressing varicosities decreases glutamatergic synaptic efficacy onto PV-INs in the NAcSh (TMx PVIN: $50.60 \pm 4.39 \%, n=10, p<0.001$; Fig. $1 G, I, J)$. We next asked whether this effect simultaneously occurs at glutamatergic synapses onto MSNs, as these synapses grossly outnumber feedforward inputs onto PV-INs and are the canonical sites of neuromodulation in the NAc (Lüscher and Malenka, 2011; Manz et al., 2019). To our surprise, unlike synapses onto PV-INs, TMx had no effect on EPSC amplitude in tdT(-) MSNs [TMx MSN: $99.80 \pm 2.22 \%$, $n=7, p=0.999$, one-way repeated measures (RM)-ANOVA, TMx cell-type effect, $p<0.001, F_{(3,30)}=14.15$, Sidak's post hoc analysis; Fig. $1 H-J]$. To circumvent the pharmacological contingency on NET expression with TMx, we incorporated NE (1-10 $\mu \mathrm{M})$ into the ACSF bath while recording EPSCs in tdT(-) MSNs and $\operatorname{tdT}(+) \mathrm{PV}$-INs. Congruent with experiments performed with TMx and desipramine, NE significantly decreased EPSC amplitude in PV-INs (NE PV-IN: 66.17 $\pm 6.06 \%, n=6$, $p<0.001$; Fig. $1 K, M, N)$. However, on average, NE had no discernible effect at synapses onto MSNs (NE MSN: $96.79 \pm 2.69 \%$, $n=7, p=0.887$, one-way RM-ANOVA, NE cell-type effect, $p<0.001, F_{(3,22)}=4.115$, Sidak's post hoc analysis; Fig. $\left.1 L-N\right)$. Thus, glutamatergic synapses onto PV-INs and MSNs, despite arising from the same corticolimbic and thalamic structures, are differentially regulated by NE signaling in the NAcSh. 

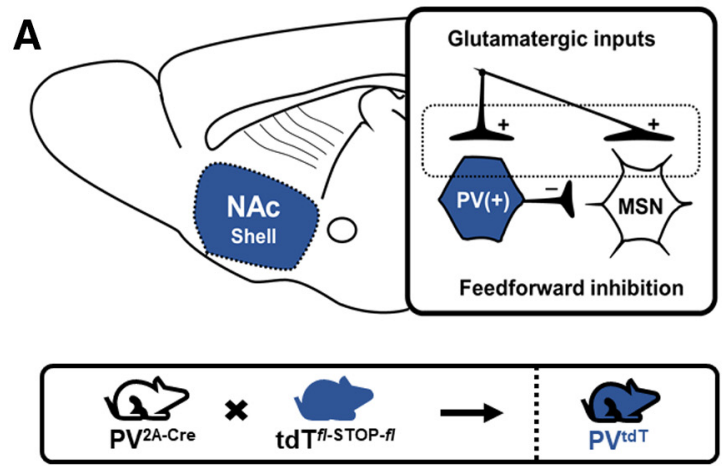

B
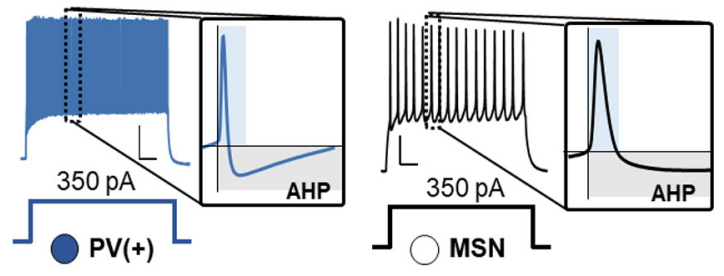

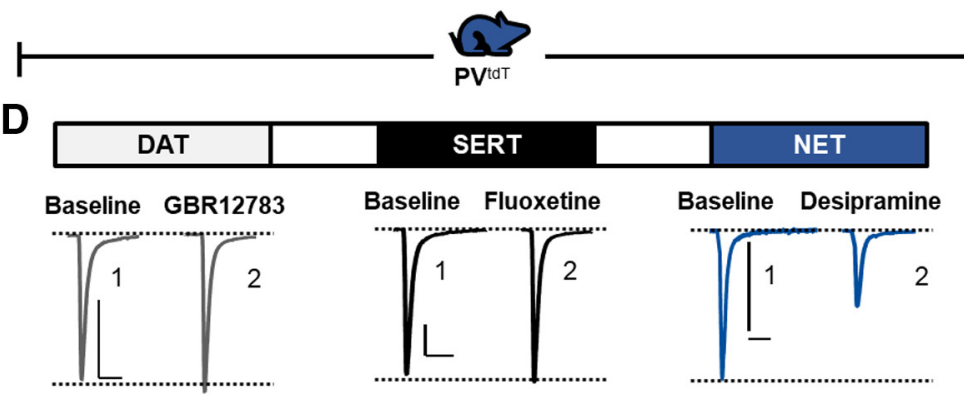

C

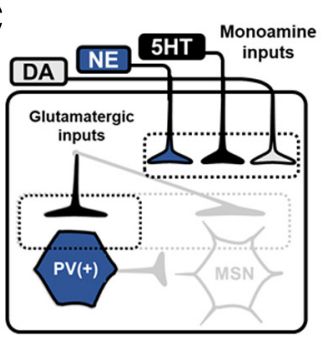

E
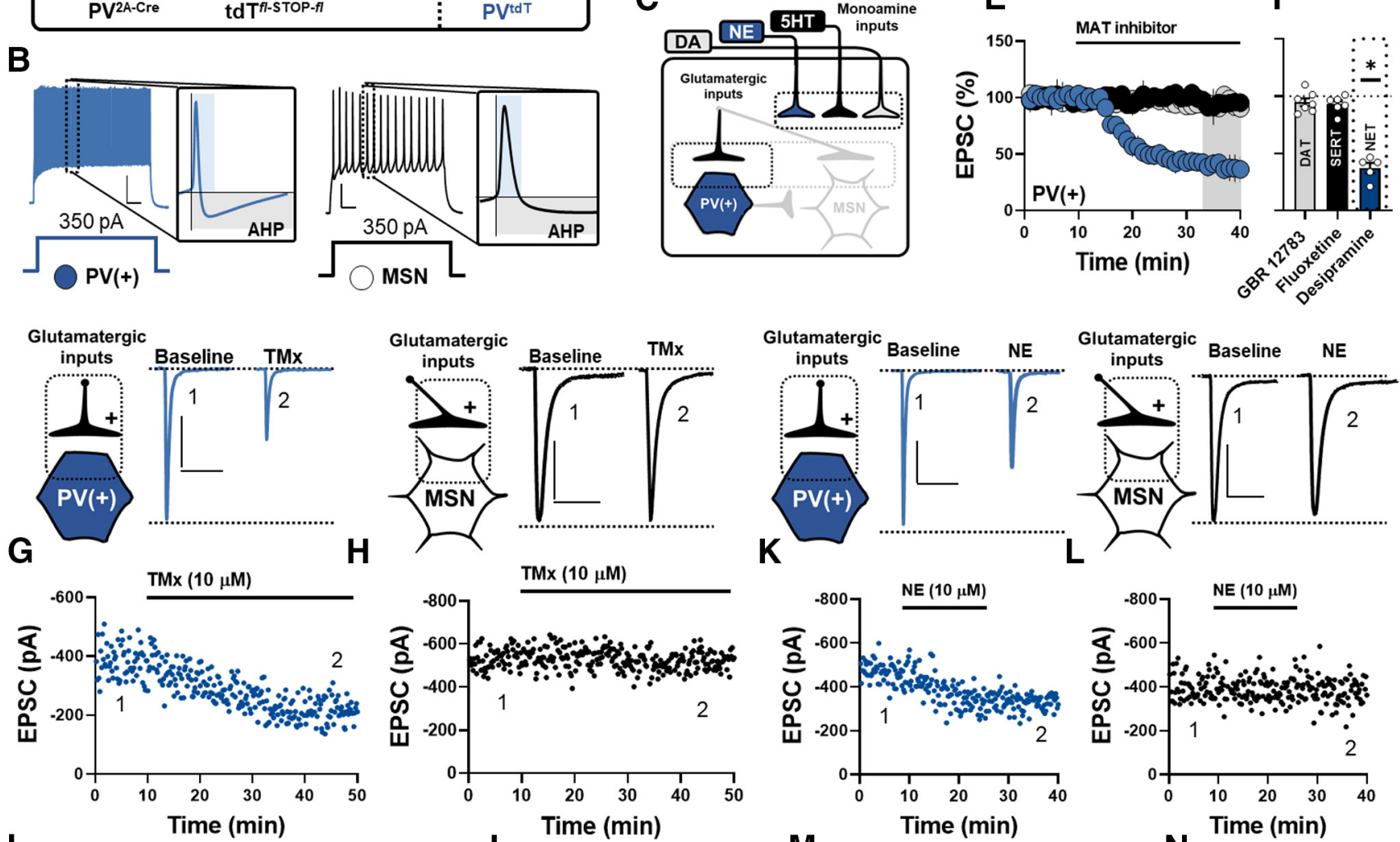

I
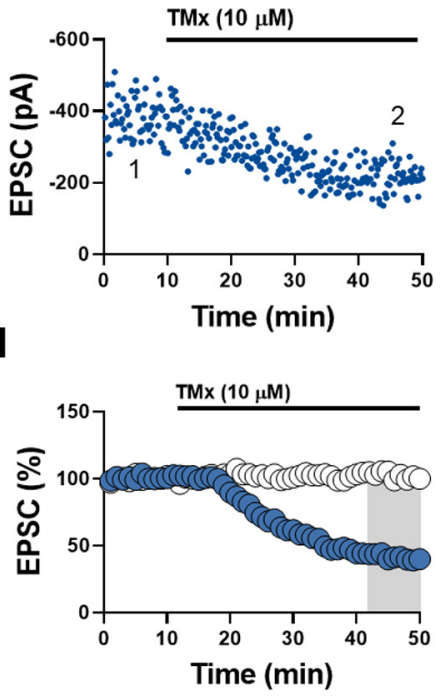
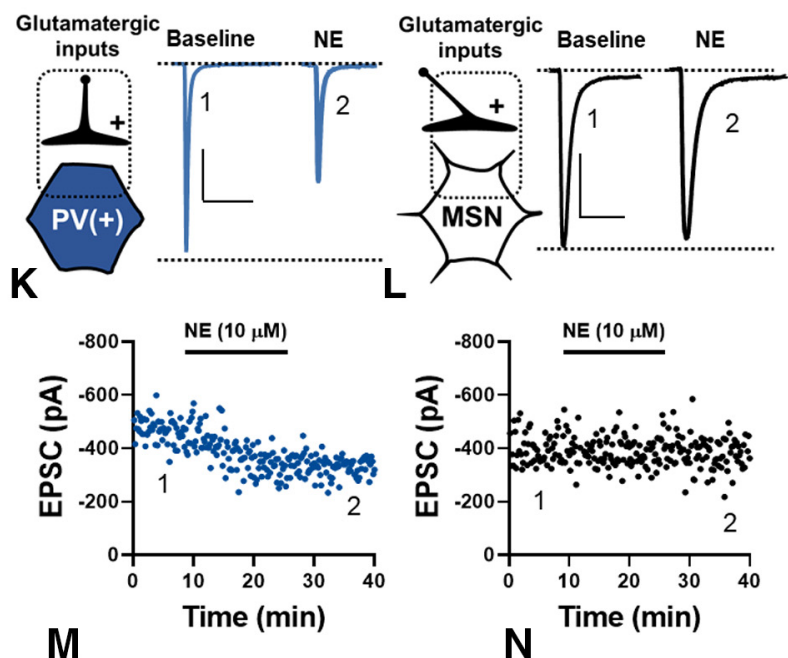

N

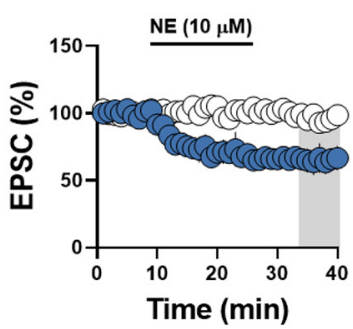

Figure 1. NET blockade decreases glutamatergic drive onto fast-spiking PV-INs but not MSNs in the NAcSh. $\boldsymbol{A}$, top, Parasagittal mouse brain slice depicting recording region within NAcSh and the PV-IN-containing feedforward inhibitory microcircuit Bottom, Schematic depicting breeding strategy used to generate PV ${ }^{\text {Cre }}$-tdTomato ${ }^{\text {fl-sToP-fl }}$ (PV ${ }^{\text {tdT }}$ ) mice. $\boldsymbol{B}$, left, Representative traces of APs elicited via 350-pA somatic current injection in tdT(+) PV-INs and tdT(-) MSNs. Scale bar: $20 \mathrm{mV} / 100 \mathrm{~ms}$. C, Schematic of candidate monoaminergic inputs regulating feedforward glutamatergic synapses onto PV-INs. D, Representative traces of EPSCs obtained preapplication and postapplication of DAT inhibitor, GBR12783 (gray), SERT inhibitor, fluoxetine (black), or tricyclic NET inhibitor, desipramine (blue). Scale bars: 100 pA/20 ms. $\boldsymbol{E}$, Time course summary of EPSCs in tdT(+) PV-INs cells during application of each MAT inhibitor. $\boldsymbol{F}$, Quantification of average EPSCs amplitude post-MAT inhibitor at $t$ (gray) $=35-40$. G, Representative experiment and traces of EPSCs in tdT(+) PV-INs during bath-application of the more selective NET inhibitor, TMx. $\boldsymbol{H}$, Representative experiment and traces of EPSCs in tdT(-) MSNs during bath-application of TMx. $\boldsymbol{I}$, Time course of EPSCs obtained from tdT(+) PV-INs and tdT(-) MSNs during TMx application. $J$, Quantification of average EPSCs before and after $[t($ gray $)=45-50]$ TMx application in tdT(+) PV-INs and tdT(-) MSNs. $K$, Representative experiment and traces of EPSCs in tdT(+) PVINs during bath-application of NE. $L$, Representative experiment and traces of EPSCs in $\mathrm{tdT}(-)$ MSNs during bath-application of NE. $M$, Time course summary of EPSCs obtained from tdT(+) PV-INs and tdT(-) MSNs during NE application. N, Summary of average EPSCs before and after [t(gray) $=45-50]$ NE application in tdT(+) PV-INs and tdT(-) MSNs. All scale bars: 100 pA/50 ms. Error bars indicate SEM; $* p<0.05$. 
Postsynaptic $\alpha_{2}$-ARs mediate the actions of NE signaling at glutamatergic synapses onto PV-INs

While the NAc is only sparsely innervated by $\mathrm{NE}$ afferents, $\alpha$-AR and $\beta$-AR are expressed highly in both the NAc core and shell with differential effects on downstream signaling pathways (Kirouac and Ciriello, 1997; McKittrick and Abercrombie, 2007; Fig. 2A). $\beta$-ARs $(\beta 1-\beta 3)$ are collectively $\mathrm{G}_{\mathrm{s}}$-coupled $\mathrm{G}$-protein-coupled receptors (GPCRs), whereas $\alpha_{1}$-AR and $\alpha_{2}$-AR are $\mathrm{G}_{\mathrm{q}}$-coupled and $\mathrm{G}_{\mathrm{i} / \mathrm{o}}$-coupled GPCRs, respectively (Federman et al., 1992). To identify which AR subtype mediates the effect of NE signaling at glutamatergic synapses onto PV-INs, we first superfused TMx in the presence of $\beta$-AR antagonist, propranolol $(5 \mu \mathrm{M})$. The TMx-induced depression in EPSC amplitude remained completely intact in the presence of propranolol (TMx in PROP: $53.22 \pm 3.65 \%, \quad n=10, \quad p<$ 0.001; Fig. 2B,C,E). However, in ACSF containing pan- $\alpha$-AR antagonist, phentolamine $(1 \mu \mathrm{M})$, TMx had no significant effect on EPSC amplitude, indicating that NET-regulated NE release decreases glutamatergic transmission onto PV-INs via $\alpha$-ARs (TMx in phentolamine: $97.83 \pm 2.99 \%, n=6$, $p=0.997$; Fig. $2 B, C, E)$. We next examined the contribution of $\alpha_{2}$-ARs to the effect of TMx, as prior studies suggest that $\alpha_{2}$-AR activity decreases glutamatergic transmission in the NAc (Peng et al., 2018). In the presence of $\alpha_{2}$-AR-selective antagonist, atipamezole $(1 \mu \mathrm{M})$, the TMxinduced decrease in EPSC amplitude was completely abolished (TMx in atipamezole: $93.27 \pm 4.01 \%, n=6, p=0.588$; Fig. $2 B, D$, $E)$. Furthermore, atipamezole reversed the NE-induced depression in EPSC amplitude, suggesting that the acute inhibitory actions of NE signaling are $\alpha_{2}$-AR dependent (NEatipamezole, $98.76 \pm 5.54 \%, \quad n=5$, $p=0.003$; Fig. $2 F-H)$. To further characterize $\alpha_{1}$-AR and $\alpha_{2}$-AR function at glutamatergic synapses onto PV-INs, we bathapplied selective $\alpha_{1}$-AR and $\alpha_{2 \mathrm{~A}}$-AR agonists, $\mathrm{PE}(1 \mu \mathrm{M})$ and guanfacine $(5 \mu \mathrm{M})$, respectively. Consistent with the $\alpha_{2}$-AR-dependent actions of TMx and NE, guanfacine resulted in a robust depression in EPSC amplitude, whereas $\mathrm{PE}$ had no effect (PE, $97.05 \pm 1.46 \%, n=6$; guanfacine, $54.51 \pm$ $5.15 \%, \quad n=9, \quad p<0.001$; Fig. $2 I-K)$. Mirroring the synapse-specific effects of NE and TMx at PV-INs, guanfacine had minimal effect on EPSC amplitude in MSNs (GUAN, tdT(-) MSN local EPSCs, $93.02 \pm 7.16 \%, n=7, p=0.349$; Fig. $2 L-N)$. Collectively, these data indicate that $\mathrm{NE}$
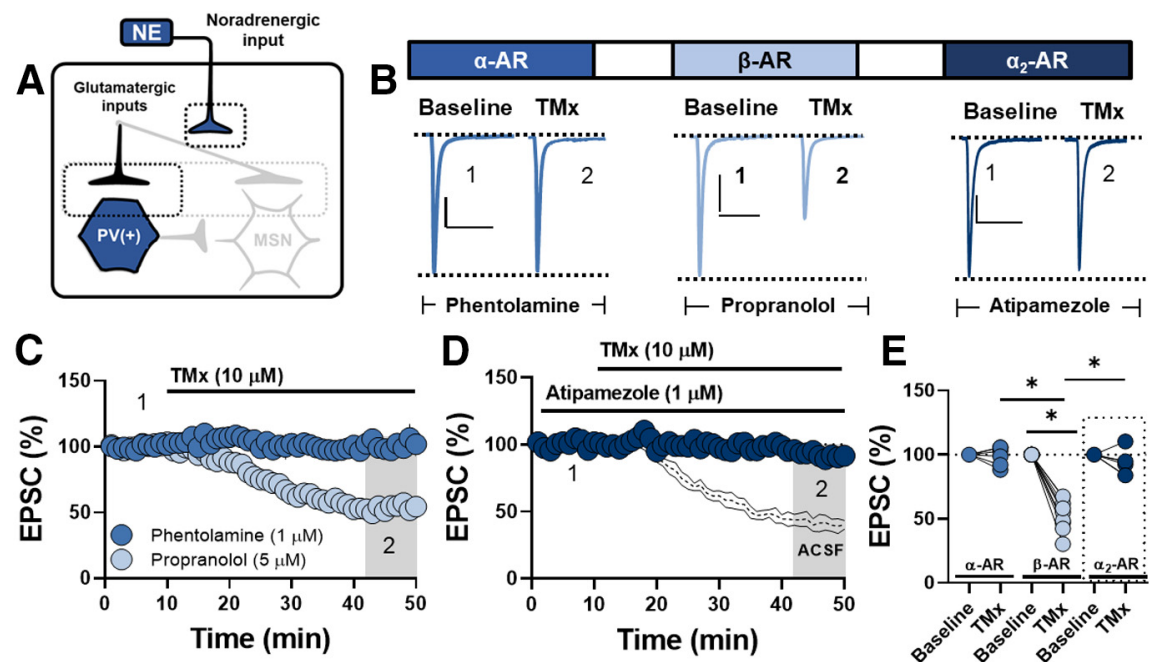

$\mathbf{F}$
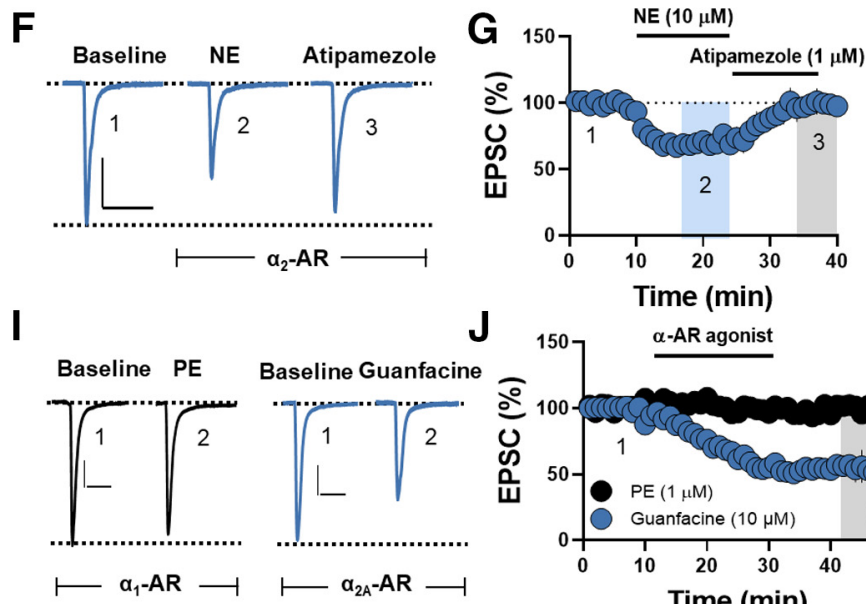

J
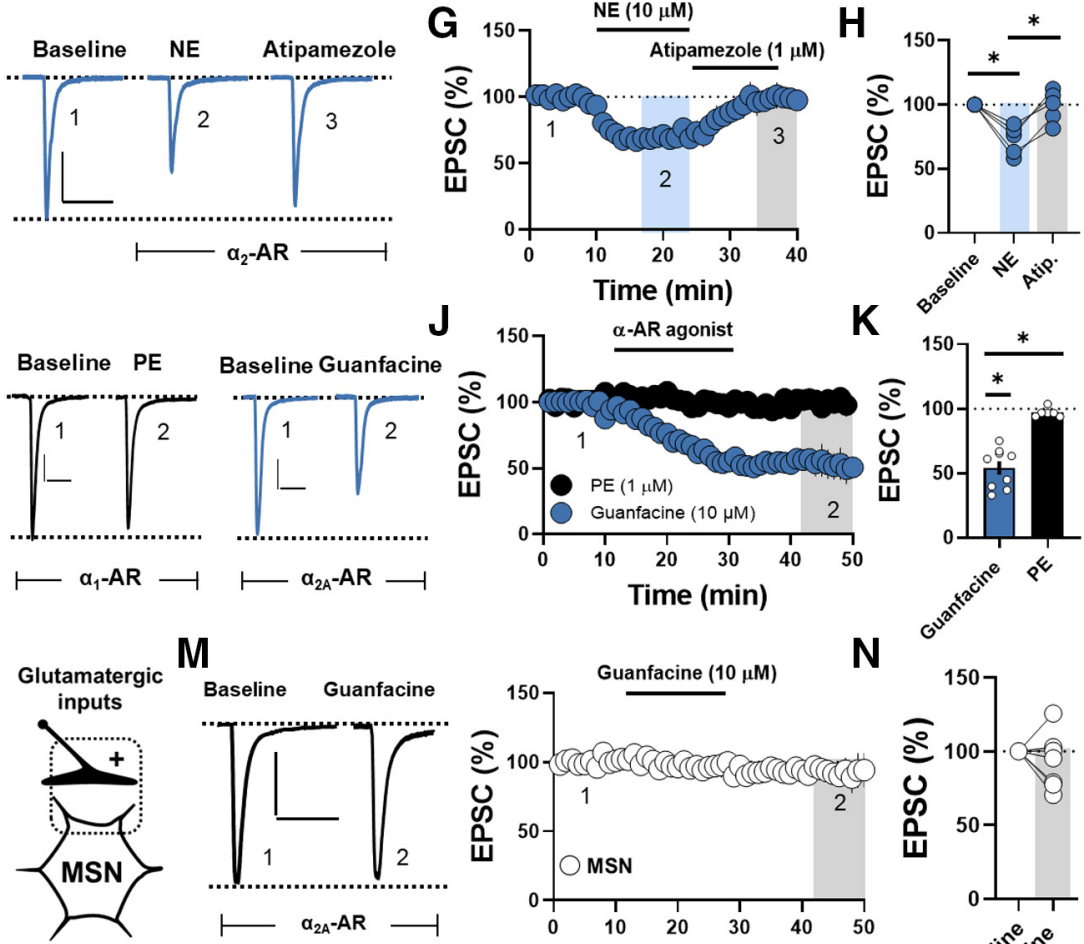

M
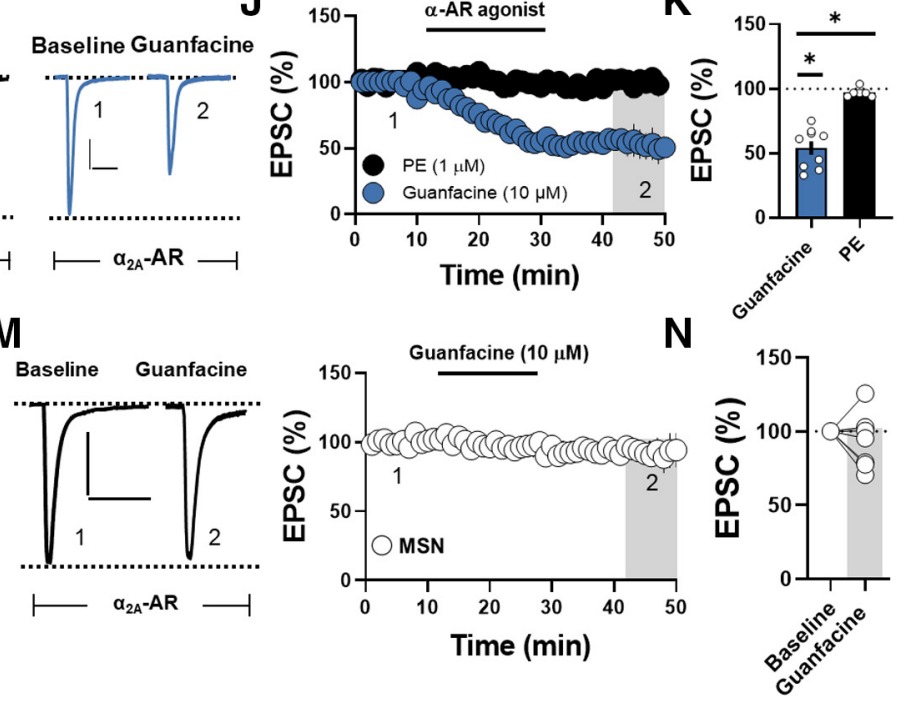

Figure 2. $\quad \alpha_{2}$-ARs mediate the actions of NE signaling at glutamatergic synapses onto PV-INs. $\boldsymbol{A}$, Schematic depicting NE input regulating PV-IN-embedded feedforward inhibitory microcircuit. $\boldsymbol{B}$, Representative traces of EPSCs in tdT(+) PV-INs during bath-application of TMx in ACSF containing non-selective $\alpha$-AR antagonist, phentolamine (left); non-selective $\beta$-AR antagonist, propranolol (middle); and selective $\alpha_{2}$-AR antagonist, atipamezole (right). Scale bars: $100 \mathrm{pA} / 50 \mathrm{~ms}$. C, Time course summary of EPSCS obtained from tdT(+) PV-INs during bath-application of TMx in ACSF containing non-selective phentolamine (dark blue) or propranolol (light blue). D, Time course summary of EPSCs obtained from tdT(+) PV-INs during bath-application of TMx in ACSF containing atipamezole. Gray-shaded region depicts the ghosted ACSF control. $\boldsymbol{E}$, Quantification of average EPSCs in tdT(+) PV-INs before and after [t(gray) $=45-50]$ TMx application in the presence of each AR antagonist. $\boldsymbol{F}$, Representative traces of EPSCs in $\mathrm{tdT}(+)$ PV-INs at baseline, during NE superfusion, and in the presence of atipamezole. Scale bars: $100 \mathrm{pA} / 50 \mathrm{~ms}$. G, Time course summary of EPSCs obtained from tdT(+) PV-INs during bath-application of NE chased by atipamezole. $\boldsymbol{H}$, Quantification of average EPSCs following NE superfusion [t(blue) $=15-$ $35]$ and in the presence of atipamezole $[t($ gray $)=35-40 \mathrm{~min}]$. $I$, Representative traces of EPSCs in $\operatorname{tdT}(+)$ PV-INs before and after PE or guanfacine. Scale bars: $100 \mathrm{pA} / 20 \mathrm{~ms}$. J, Time course summary of EPSCs obtained from tdT(+) PV-INs during bath-application of $\alpha_{1}$-AR agonist, PE (black) or $\alpha_{2 A}$ AR agonist, guanfacine (blue). $\boldsymbol{K}$, Quantification of average EPSCs following bath-application of PE or guanfacine. $L$, left, Schematic depicting MSN recording strategy within feedforward inhibitory microcircuits. $\boldsymbol{M}$, Representative EPSCs and time course summary of electrically-evoked local EPSCs in $\operatorname{tdT}(-)$ MSNs during bath-application of guanfacine. $\boldsymbol{N}$, Quantification of average EPSC amplitude postguanfacine in MSNs. Scale bar: 100 $\mathrm{pA} / 50 \mathrm{~ms}$. Error bars indicate SEM; $* p<0.05$. 
A
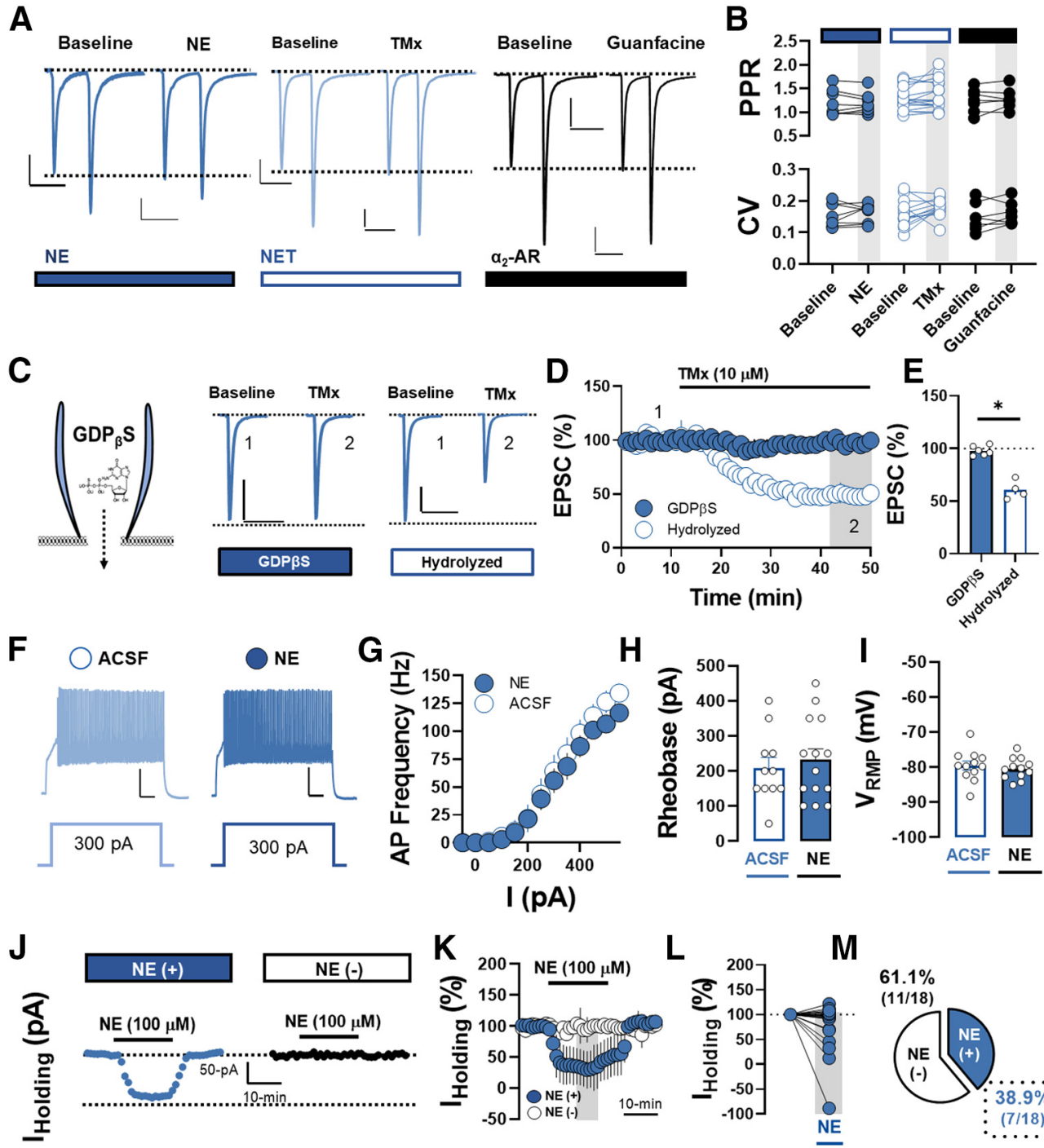

M

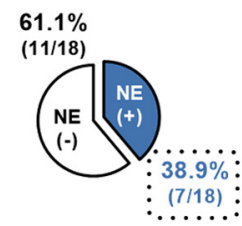

Figure 3. NE signaling is mediated by a postsynaptic mechanism at glutamatergic synapses onto PV-INs. $\boldsymbol{A}$, Representative traces of 50-ms ISI paired-pulse EPSCS at baseline and in the presence of NE (blue), TMx (light blue), and guanfacine (black). Scale bars: 100 pA/50 ms. B, Quantification of average PPR and CV pre-NE and post-NE, TMx, and guanfacine. C, Representative traces of EPSCs in tdT(+) PV-INs during bath-application of TMx with $\mathrm{GDP}_{\beta}$ S-loaded internal solution (left) and a thermally-hydrolyzed control internal solution (right). Scale bars: $100 \mathrm{pA} / 50$ ms. $\boldsymbol{D}$, time course summary of EPSCs obtained from tdT(+) PV-INs during bath-application of TMx with GDP ${ }_{\beta}$ S-loaded internal solution (dark) and a thermally hydrolyzed control internal solution (open circles). $E$, Quantification of average EPSCs post-TMx $\left[t(\right.$ gray) $=45-50 \mathrm{~min}]$ in $\mathrm{GDP}_{\beta} S$ and control internal solutions. $\boldsymbol{F}$, Representative traces of APs elicited via 300-pA somatic current injection in ACSF (light blue) or NE (dark blue). Scale bar: $20 \mathrm{mV} / 100 \mathrm{~ms}$. G, Input-output function of PV-INs following 50-pA sequential increases in current injection (ACSF, open circles; NE, blue circles). $\boldsymbol{H}$, Rheobase obtained from PV-INs in ACSF or NE. $I, V_{\text {RMP }}$ of PV-INs in ACSF or NE. J, left, Representative experiment of an NE-induced shift in $I_{\text {Holding, }}$ rendering the cell NE(+) (blue). Right, Representative experiment of a PV-IN not undergoing a change in $I_{\text {Holding, }}$ rendering the cell NE(-) (black). Scale bar: $50 \mathrm{pA} / 10$ min. $\boldsymbol{K}$, Segregated time course summary depicting the effects of NE on $I_{\text {Holding }}$ in NE(+) (blue) and NE(-) (open circles) PV-INs. L, Quantification of $I_{\text {Holding }}$ post-NE in all 18 PV-INs quantified at $t($ gray) $=20$ min. $M$, Pie chart summary showing

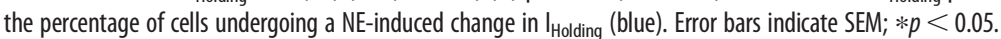

signaling via $\alpha_{2}$-ARs selectively decreases glutamatergic transmission onto PV-INs.

Heterosynaptic $\alpha$-ARs are expressed on presynaptic afferents in the NAcSh, with early reports implicating an $\alpha_{2}$-AR-dependent effect on glutamate release probability (Nicola and Malenka, 1998; Peng et al., 2018). To define the synaptic locus recruited by $\mathrm{NE}$ signaling at glutamatergic synapses onto PV-INs, we first examined changes in the PPR and CV of EPSCs following multiple pharmacological manipulations. Bath-application of TMx or NE did not result in a change in PPR and CV (PPR = ACSF: $1.20 \pm 0.09$, NE: $1.18 \pm 0.08, n=8, p=0.6414$; ACSF: $1.29 \pm 0.06$, TMx: $1.34 \pm 0.076, n=17, p=0.657$; CV $=$ ACSF: $0.15 \pm 0.01, \mathrm{NE}$ : $0.16 \pm 0.01, \quad n=8, \quad p=0.4=5477 ;$ ACSF: $0.16 \pm 0.013, \quad \mathrm{TMx}$ : $0.18 \pm 0.01, n=12, p=0.186$; Fig. $3 A, B)$. Similarly, $\alpha_{2 \mathrm{~A}}$-AR agonist, guanfacine, had no effect on PPR or CV, suggesting that presynaptic glutamate release probability is unaffected by NE signaling (PPR = ACSF: $1.25 \pm 0.09$, GUAN: $1.28 \pm 0.08, n=7$, $p=0.4972 ; \mathrm{CV}=\mathrm{ACSF}: 0.15 \pm 0.017$, GUAN: $0.16 \pm 0.014$, $n=7, p=0.9830$; Fig. $3 A, B)$.

If NE effector function is restricted to postsynaptic domains, disabling postsynaptic G-protein signaling should also abolish the effects of NE transmission. Incorporation of cell-impermeant GDP analog, GDP ${ }_{\beta} S(1 \mathrm{~mm})$, into the intracellular solution of the patch pipette completely blocked the TMx-induced depression in EPSC amplitude relative to a thermally-hydrolyzed control solution $\left(\mathrm{GDP}_{\beta} \mathrm{S}, 97.96 \pm 1.88 \%, n=6\right.$; control, $60.80 \pm 4.44 \%$, $n=4, p<0.001$; Fig. $3 G-I)$.

To assess whether the postsynaptic effects of NE coincide with shifts in intrinsic membrane excitability, we quantitated the input-output function of PV-INs via somatic current injection in 

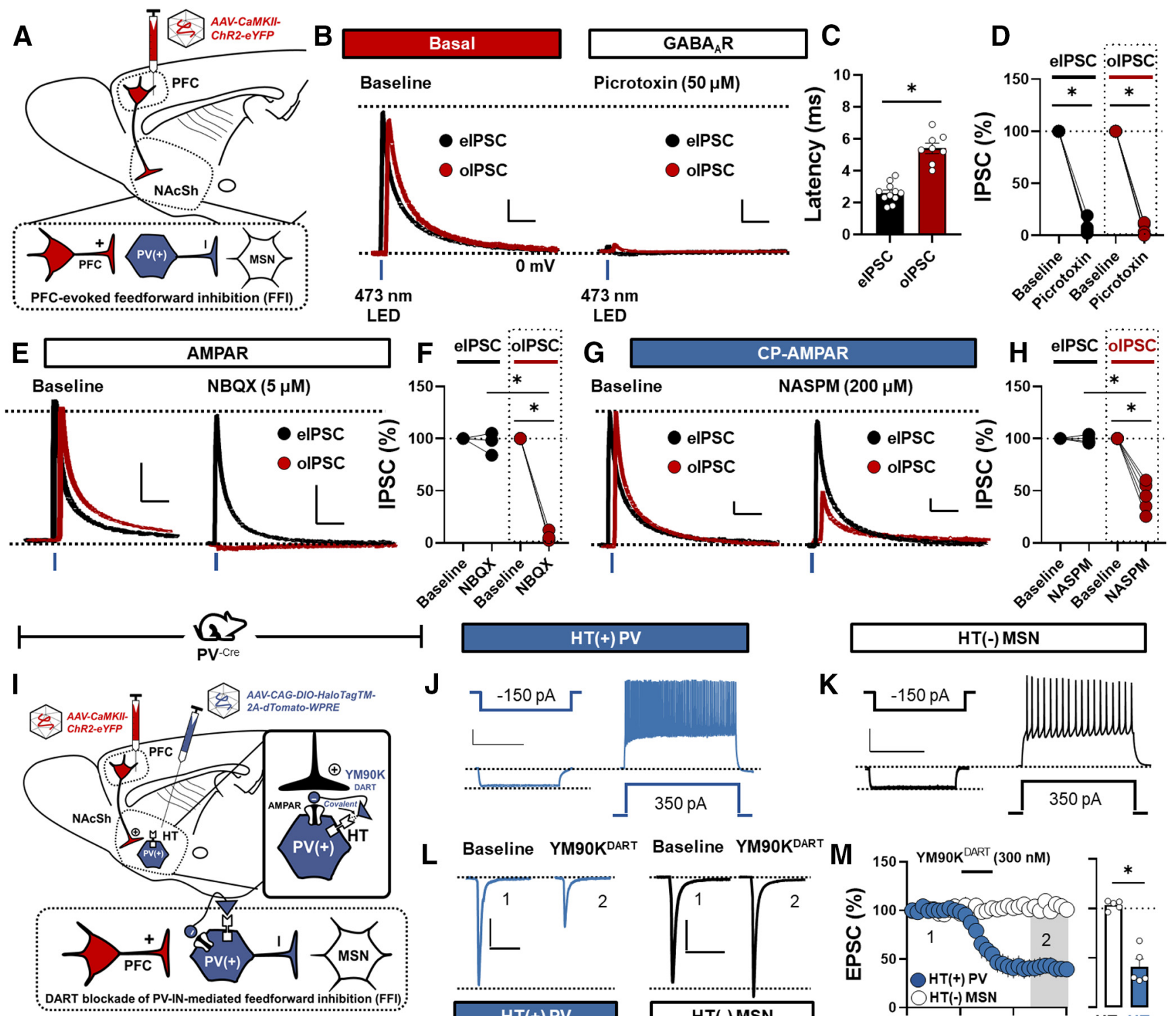

HT(+) PV

J

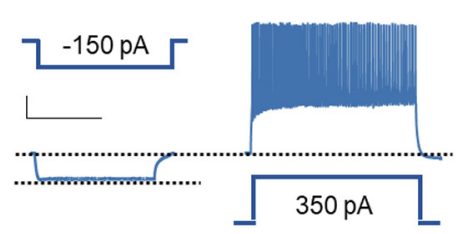

L

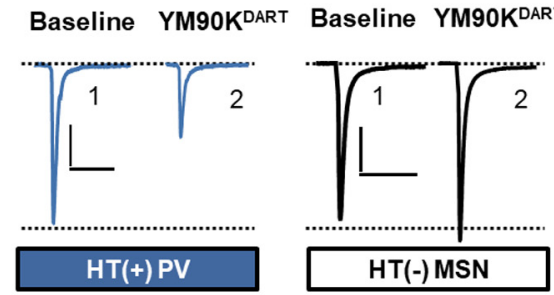

\section{HT(+) PV}

N

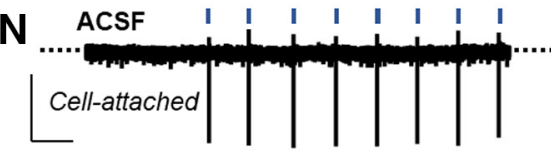

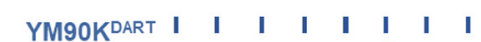

\section{0}

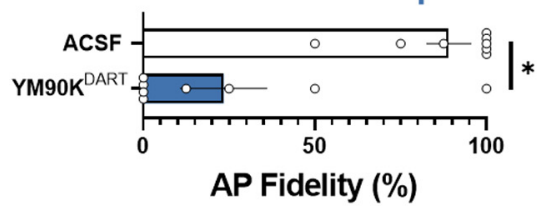

HT(-) MSN

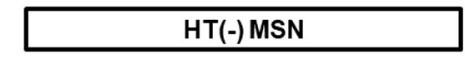

K

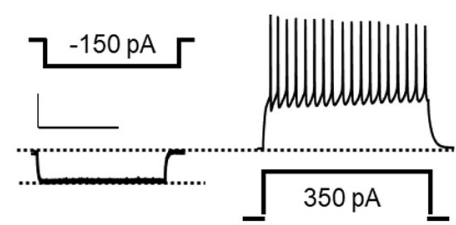

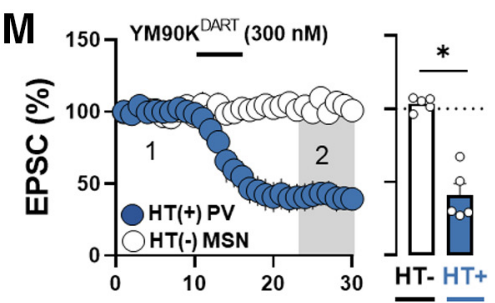

Time (min)

\section{PV-IN-specific AMPAR}

$\mathbf{P}$
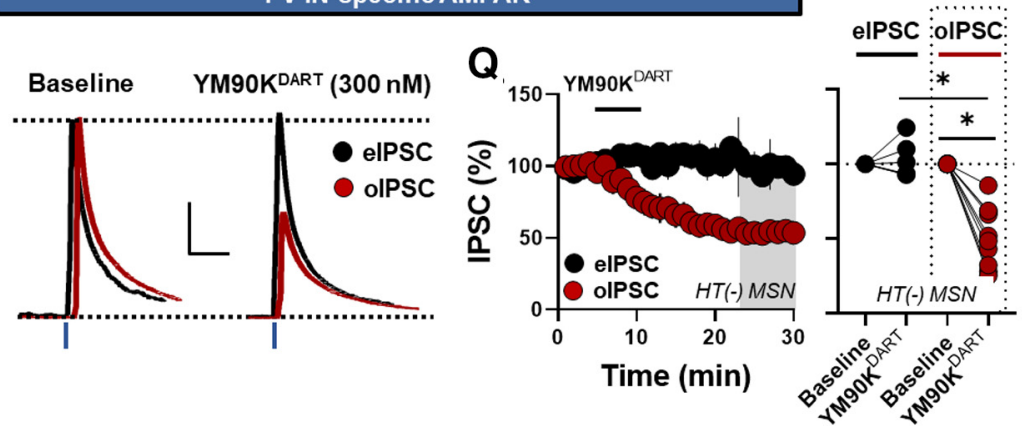

Figure 4. PV-INs mediate PFC-evoked feedforward inhibition of MSNs in the NACSh. A, Schematic depicting optogenetic targeting of PFC afferents to the NAcSh to examine PFC-evoked FFI in WT mice. $\boldsymbol{B}$, left, Representative traces of PFC-evoked oIPSCS (red) and local elPSCS (black) obtained from MSNs at $0 \mathrm{mV}$ in ACSF. Right, Representative traces and quantification of PFCevoked olPSCs and local elPSCs obtained from MSNs in ACSF containing GABA R antagonist, picrotoxin. C, Quantification of latency onset of elPSCS and olPSCs in MSNs. D, Quantification of average e/oIPSC amplitude postpicrotoxin. $\boldsymbol{E}$, Representative traces of PFC-evoked oIPSCs and local elPSCs obtained from MSNs at baseline and in ACSF containing pan-AMPAR antagonist, NBQX. $\boldsymbol{F}$, Quantification of average oIPSC and eIPSC amplitude pre-NBQX and post-NBQX. G, Representative traces of olPSCs and eIPSCs at baseline and in the presence of CP-AMPAR antagonist, NASPM. $\boldsymbol{H}$, Quantification of average oIPSC and elPSC amplitude pre-NASPM and post-NASPM. All IPSC scale bars: $50 \mathrm{pA} / 50 \mathrm{~ms}$. I, Schematic depicting the use of DART pharmacology to selectively block AMPAR-mediated feedforward glutamatergic transmission onto PV-INs in the NAc. J, Representative traces of Tom/HT(+) PV-IN membrane responses during hyperpolarizing $(-150 \mathrm{pA})$ and depolarizing current steps (350 pA). Scale bar: $20 \mathrm{mV} / 500 \mathrm{~ms}$. $\boldsymbol{K}$, Representative traces of Tom/HT(-) MSN membrane responses during hyperpolarizing (-150 pA) and depolarizing current 
slices containing NE $(10 \mu \mathrm{M})$ or ACSF alone. No significance differences were observed in AP firing, rheobase, or resting membrane potential $\left(\mathrm{V}_{\mathrm{RMP}}\right)$ between conditions (AP frequency $500 / 300-$ pA ACSF: $126.39 \pm 9.92 \mathrm{~Hz} / 64.03 \pm 14.54 \mathrm{~Hz}, n=9$; AP frequency $300 / 500-p A$ NE: $106.46 \pm 5.92 \mathrm{~Hz} / 55.74 \pm 10.81 \mathrm{~Hz}$, $n=14$, one-way RM-ANOVA, $p=0.0700 / 0.4319$; Rheobase = ACSF: $209.1 \pm 30.01 \mathrm{pA}, n=11$; NE: $232.1 \pm 31.28 \mathrm{pA}, n=14$, $p=0.6072 ; \quad \mathrm{V}_{\mathrm{RMP}}=$ ACSF: $-79.57 \pm 1.25 \mathrm{mV}, n=12 ; \quad \mathrm{NE}:$ $-80.44 \pm 0.88 \mathrm{mV}, n=12, p=0.5045$; Fig. $3 J-M)$. In contrast to these characterizations of physiological NE signaling, application of a supraphysiological concentration of NE $(100 \mu \mathrm{M})$ was occasionally accompanied by a decrease in holding current $\left(\mathrm{I}_{\text {Holding }}\right)$ and membrane resistance $\left(\mathrm{R}_{\mathrm{m}}\right.$, data not shown) that returned to baseline following drug washout. The concomitant reduction in $\mathrm{R}_{\mathrm{m}}$ and $\mathrm{I}_{\text {Holding }}$ likely points to a NE-evoked inward current, which was appreciable in only a fraction of cells $[\mathrm{NE}(+)]\left(\mathrm{I}_{\text {Holding }}\right.$ NE, all cells: $50.53 \pm 11.84 \%, n=7 / 18$ cells, $p=0.0540$; Fig. $3 C-F)$. Together, these data provide functional evidence that $\mathrm{NE}$ decreases glutamatergic transmission onto $\mathrm{PV}(+)$-INs by recruiting postsynaptic $\alpha_{2}$-AR without altering presynaptic glutamate release.

\section{$\alpha_{2 \mathrm{~A}}$-AR function at PV-in synapses diminishes PFC-evoked feedforward inhibition onto MSNs in the NAcSh}

Our data indicate that glutamatergic inputs onto PV-INs are under privileged neuromodulatory control by NE signaling, suggestive of a novel motif for disengaging feedforward inhibition (FFI). To examine this idea, we employed a dynamic whole-cell patch-clamp electrophysiological approach in mice expressing channelrhodopsin-2 (ChR2) in the medial PFC (Fig. 4A). To isolate PFC-evoked FFI onto MSNs, we used a $\mathrm{Cs}^{+}$-based intracellular solution with $\mathrm{E}_{\mathrm{Cl}}$ and $\mathrm{E}_{\mathrm{AMPAR} / \mathrm{NMDAR}}$ calibrated to approximately -80 and $0 \mathrm{mV}$, respectively. At $0 \pm 5 \mathrm{mV}$, electrically-evoked IPSCs (eIPSCs) were interleaved with opticallyevoked IPSCs (oIPSCs) from the PFC (Fig. 4B). oIPSCs exhibited increased latency relative to interleaved eIPSCs, indicating the presence of a polysynaptic feedforward circuit (latency, eIPSCs: $2.61 \pm 0.18 \mathrm{~ms}, n=11$; oIPSCs: $5.40 \pm 0.33 \mathrm{~ms}, n=8, p<0.001$; Fig. $4 B, C)$. We first confirmed that IPSCs obtained at $0 \mathrm{mV}$ were mediated by $\mathrm{GABA}_{\mathrm{A}} \mathrm{Rs}$ by superfusing picrotoxin $(50 \mu \mathrm{M})$ into the ACSF bath. Picrotoxin completely blocked eIPSC and oIPSC amplitude, confirming that $\mathrm{GABA}_{\mathrm{A}}$ Rs mediate PFC-evoked and locally-evoked GABAergic transmission onto MSNs at $\sim 0 \mathrm{mV}$ (PTX, eIPSCs: $7.78 \pm 3.01 \%, n=5, p<0.001$; oIPSCs: $6.04 \pm$ $2.39 \%, n=5, p<0.001$, one-way ANOVA, electrical vs optical effect, $F_{(3,16)}=2.50, p=0.919$; Fig. $\left.4 B, D\right)$. If PFC-evoked oIPSCs in MSNs are mediated by disynaptic FFI, bath-application of pan-AMPAR antagonist, NBQX $(5 \mu \mathrm{M})$, should selectively eliminate oIPSCs at $0 \mathrm{mV}$. Indeed, NBQX blocked oIPSC amplitude without no impact on locally-evoked monosynaptic eIPSCs,

$\leftarrow$

steps (350 pA). Scale bar: $20 \mathrm{mV} / 500 \mathrm{~ms}$. L, Representative EPSCs from Tom/HT(+) PV-INs (blue) and Tom/HT(-) MSNs (black) preapplication and postapplication of AMPAR DART, YM90K ${ }^{\text {DART }}$. $M$, Normalized time course summary and quantification of EPSCs in Tom $/ \mathrm{HT}(+)$ PV-INs (blue) and Tom/HT(-) MSNs (open circles) during YM90K ${ }^{\text {ART }}$ superfusion. $\mathbf{N}$, Representative traces of PFC-evoked APs in PV-INs in cell-attached configuration in ACSF (black) or YM90K ${ }^{\text {DART }}$ (300 nM; blue). O, Quantification of AP fidelity (number of AP successes $\times$ eight pulses) in ACSF versus YM90K ${ }^{\text {DART }}$. Scale bar: $50 \mathrm{mV} / 2$ s. P, Representative traces of PFC-evoked olPSCs and local elPSCs obtained from Tom/HT(-) MSNs at baseline and following YM90K ${ }^{\text {DART }}$ application. Scale bar: $50 \mathrm{pA} / 50 \mathrm{~ms}$. Normalized time course summary and quantification of average IPSC amplitude pre-YM90K ${ }^{\text {DART }}$ and post-YM90K ${ }^{\text {DART }}$. Error bars indicate $\mathrm{SEM} ; * p<0.05$. indicating that oIPSCs in MSNs require AMPAR-mediated excitation of a GABAergic intermediate (NBQX, eIPSCs: $95.76 \pm 6.20 \%$, $n=3, p=0.820$; oIPSCs: $6.57 \pm 2.97 \%, n=3, p<0.001$, one-way ANOVA, electrical vs optical effect, $F_{(3,8)}=2.08, p<0.001$; Fig. $4 E, F)$.

To determine whether PV-INs are the primary GABAergic cell-type mediating FFI in the NAcSh, we took advantage of the fact that CP-AMPARs are selectively expressed at feedforward synapses onto PV-INs (Manz et al., 2020b). Following a 5-min eIPSC and oIPSC baseline, NASPM $(200 \mu \mathrm{M})$ was added to the ACSF bath. While eIPSC amplitude remained unchanged in the presence of NASPM, PFC-evoked oIPSC amplitude was significantly reduced, indicating that a CP-AMPAR-expressing GABAergic IN mediates FFI of MSNs in the NAcSh (NASPM, eIPSCs: $99.24 \pm 1.87 \%, n=4, p=0.990$; oIPSCs: $43.99 \pm 6.63 \%$, $n=5, p<0.001$, one-way ANOVA, electrical vs optical effect, $F_{(3,14)}=8.03, p<0.001$; Fig. $\left.4 G, H\right)$. To further resolve the contribution of PV-INs to FFI of MSNs, we leveraged DART, a recently developed method for targeting drugs to defined celltypes (Shields et al., 2017). A Cre-dependent virus encoding a surface HaloTag (HT) protein and dTomato (Tom) was injected into the NAcSh of $\mathrm{PV}^{\mathrm{Cre}}$ mice. Tom/HT( + ) cells exhibited the defining electrophysiological features of PV-INs, including a fast-spiking, non-accommodating AP profile with no hyperpolarization-activated voltage sag, indicating that Tom/HT $(+)$ cells are PV-INs. In contrast, the vast majority of Tom/HT(-) cells exhibited no voltage sag with a regular-spiking AP profile, consistent with the properties of MSNs (Fig. 4J,K; Kawaguchi, 1993; Manz et al., 2020b). Leveraging YM90K ${ }^{\text {DART }}$, a two-headed ligand with a targeting moiety opposite an AMPAR antagonist, we confirmed that YM90K ${ }^{\text {DART }}$ does not impact EPSCs onto HT(-) neurons at an ambient concentration of $300 \mathrm{~nm}$ but accumulates within minutes on $\mathrm{HT}(+)$ neurons to elicit a robust decrease in EPSCs amplitude [HT(-) MSN YM90K ${ }^{\mathrm{DART}}: 103.4 \pm$ $2.07 \%, n=5 ; \mathrm{HT}(+)$ PV YM90K ${ }^{\mathrm{DART}}: 40.89 \pm 7.86 \%, n=5$, $p<0.001$; Fig. $4 L, M]$. Tethering of YM90K ${ }^{\mathrm{DART}}$ onto $\mathrm{HT}(+)$ neurons is covalent and persists following washout.

We next combined DART with the optogenetic FFI assay by injecting ChR2 in the PFC and the HT/Tom-expressing virus in the NAcSh of $\mathrm{PV}^{\mathrm{Cre}}$ mice. Optical stimulation of PFC afferents (one pulse, 1-ms duration, 50\% stimulation intensity) evoked high-fidelity APs in $\mathrm{HT}(+)$ PV-INs recorded in cell-attached configuration that was decreased by YM90K ${ }^{\mathrm{DART}}$ [300 nM; AP fidelity (spikes/eight pulses) in ACSF: $89.06 \pm 6.44 \%, n=8$; in YM90K $^{\text {DART }}: 23.44 \pm 12.60 \%, n=8, p=0.004$; Fig. $\left.4 N, O\right]$. In whole-cell configuration, YM90K ${ }^{\mathrm{DART}}(300 \mathrm{nM})$ significantly attenuated PFC-evoked oIPSCs but not eIPSCs from HT(-) MSNs at $0 \mathrm{mV}$, supporting our findings and others that PV-INs mediate the bulk of FFI of MSNs in the NAcSh $\left(\mathrm{YM} 90 \mathrm{~K}^{\mathrm{DART}}\right.$ eIPSCs: $102.40 \pm 5.15 \%, n=6$; YM90K ${ }^{\mathrm{DART}}$ oIPSCs: $51.82 \pm$ $6.36 \%, n=10, p<0.001$; one-way ANOVA, electrical vs optical effect, $F_{(3,28)}=34.57, p<0.001$; Fig. $\left.4 P, Q\right)$.

To assess how selective $\alpha_{2 \mathrm{~A}}$-AR activity modulates synaptic propagation through the feedforward circuit, we bath-applied guanfacine after obtaining a 5-min baseline of interleaved eIPSCs and oIPSCs from MSNs. Guanfacine elicited a robust depression in oIPSC amplitude that was remarkably similar in magnitude to experiments performed at glutamatergic synapses onto PV-INs (oIPSCs: $54.08 \pm 7.73 \%, n=7, p=0.004$; Fig. $5 A, C, D)$. In contrast, eIPSC amplitude remained intact in the presence of guanfacine, indicating that $\alpha_{2 \mathrm{~A}}$-AR activity decreases PFC-evoked FFI without concomitantly affecting monosynaptic GABAergic transmission (GUAN, eIPSCs: $92.22 \pm 5.74 \%, n=5$, 

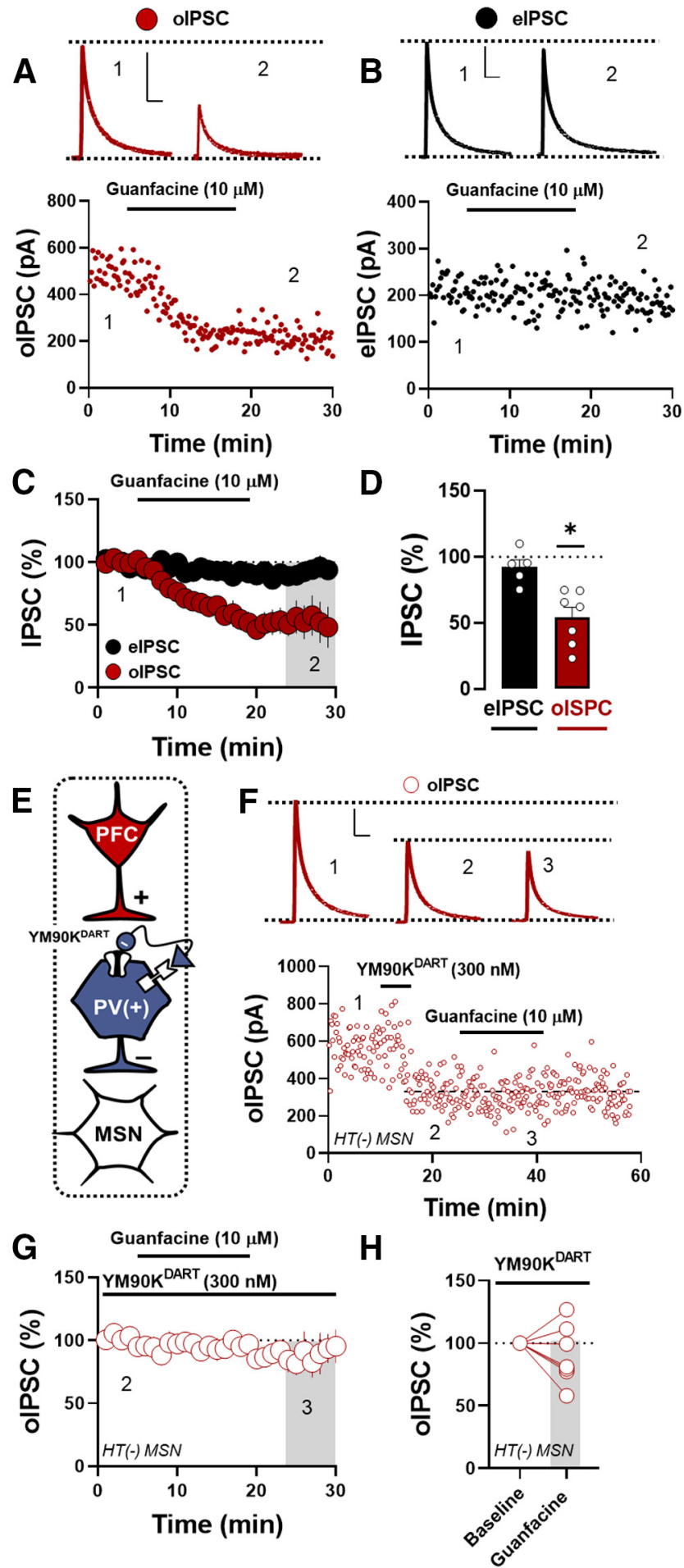

Figure 5. $\alpha_{2}$-ARs decrease PFC-evoked feedforward inhibition of MSNs in the NAcSh. $A, B$, Representative traces superimposed on experiments of PFC-evoked olPSCS and local elPSCs during bath-application of $\alpha_{2 \mathrm{~A}}$-AR agonist, guanfacine. $\boldsymbol{C}$, Time course summary of oIPSCS (red) and elPSCs (black) during bath-application of guanfacine. D, Quantification of average oIPSC and elPSC amplitude [t(gray) $=25-30 \mathrm{~min}$ ] postguanfacine. $\boldsymbol{E}$, Schematic depicting mechanism of YM90K ${ }^{\text {DRT }}$ on HT-expressing PV-INs in the NACSh. $\boldsymbol{F}$, Representative traces superimposed on experiments of PFC-evoked olPSCs in Tom/HT(-) MSNs during the sequential application of YM90K ${ }^{\text {DART }}$ (300 nM) and guanfacine. G, Renormalized time course summary of oIPSCS in the presence of YM90K DART during guanfacine superfusion. $\boldsymbol{H}$, Quantification of the average $\mathrm{YM} \mathrm{OK}{ }^{\mathrm{DART}}$-resistant oIPSC amplitude postguanfacine $[t($ gray $)=25-30 \mathrm{~min}]$. Sale bars: $50 \mathrm{pA} / 50 \mathrm{~ms}$. Error bars indicate SEM; $* p<0.05$. $p=0.781$; Fig. $5 B-D$ ). To test directly whether feedforward glutamatergic drive onto PV-INs is necessary for the effects of guanfacine, we repeated experiments using PV-IN-specific AMPAR DARTs in HT-expressing $\mathrm{PV}^{\mathrm{Cre}}$ mice (Fig. 5E). Once the YM90K ${ }^{\text {DART }}$-induced reduction in oIPSCs stabilized, guanfacine was incorporated into the ACSF bath (Fig. $5 F-H$ ). Guanfacine in the presence of YM90K ${ }^{\text {DART }}$ failed to consistently alter oIPSC amplitude in HT(-) MSNs (GUAN in YM90K ${ }^{\text {DART: }}$ $90.46 \pm 8.82 \%, n=7$; Fig. $5 G, H)$. Taken together, these findings demonstrate that NE signaling dampens feedforward transmission in the NAcSh by targeting $\alpha_{2}$-ARs at glutamatergic synapses onto PV-INs.

\section{Discussion}

By combining a dynamic electrophysiological approach with pharmacology and optogenetics in PV-IN-labeled transgenic reporter mice, we provide functional evidence that endogenous $\mathrm{NE}$ signaling imposes privileged neuromodulatory control over feedforward inhibition in the NAcSh. We report that NE signaling negatively regulates glutamatergic transmission onto PV-INs through a postsynaptic mechanism mediated by $\alpha_{2}$-ARs. At MSN synapses, however, this effect is absent, pointing to a privileged synaptic locus targeted by NE signaling in the NAcSh. Optogenetic stimulation of PFC afferents to the NAcSh revealed that $\alpha_{2}$-AR activity attenuates PV-IN-dependent feedforward inhibition of MSNs. The findings establish a microcircuit-specific regulatory module governed by a historically overlooked monoamine system in the NAcSh.

\section{NE selectively regulates glutamatergic transmission onto PV-INs}

The modest innervation pattern of NE in the NAc and robust behavioral effects of other monoaminergic systems have diverted research into how NE regulates NAc circuit function (Fallon and Moore, 1978; Brog et al., 1993; McKittrick and Abercrombie, 2007; Mitrano et al., 2012, 2018). Our initial pharmacological analysis with monoamine-specific reuptake inhibitors indicates that NET blockade is sufficient to elicit a heterosynaptic depression of glutamatergic synaptic strength onto PV-INs. In contrast, DAT blockade had no appreciable effect on EPSC amplitude at PV-IN synapses. This finding should be interpreted with caution, however, as few studies have reported a direct heterosynaptic effect of DAT blockade on glutamatergic transmission in the NAc despite extensive DAergic innervation. For example, COC, a broad-spectrum MAT inhibitor, elicits a D1 receptor-dependent decrease in glutamatergic transmission in the NAc (Nicola et al., 1996; Beurrier and Malenka, 2002; Kombian et al., 2009). Moreover, ex vivo incubation of brain slices in COC evokes DAdependent changes in glutamatergic and GABAergic synaptic strength onto MSNs (Dobbs et al., 2016; Ingebretson et al., 2018). Failure to recapitulate the TMx-induced depression in glutamatergic synaptic strength with GBR12783 is compelling evidence that DAT-regulated DA release does not exert an acute synaptic effect at glutamatergic inputs onto PV-INs.

Similar to DA, 5-HT recruits heterosynaptic mechanisms throughout the striatal network, including the NAc. For example, presynaptic $5-\mathrm{HT}_{1 \mathrm{~B}}$ receptors mediate the effects of SERT blockade and reverse 5-HT transport at glutamatergic synapses onto MSNs (Mathur et al., 2011; Heifets et al., 2019; Nardou et al., 2019). Similarly, COC-induced synaptic inhibition of ventral pallidum (VP)-projecting D2 MSNs is mediated by SERT-regulated 5-HT release but not DA (Matsui and Alvarez, 2018). We find 
that SERT blockade does not affect glutamatergic synaptic strength onto PV-INs. However, NET blockade with two structurally-distinct NET inhibitors, desipramine and TMx, evoked a robust depression in glutamatergic transmission. Interestingly, a similar finding was observed at GABAergic synapses onto NAcprojecting DA neurons in the VTA. At this VTA synapse, NE engages postsynaptically-expressed $\alpha_{1}$-ARs to promote DA neuron output, suggesting that NE signaling may serve an overall disinhibitory role on mesolimbic circuit activity (Wang et al., 2015). Furthermore, COC application elicits an $\alpha$-AR-dependent depression in synaptic strength that is occluded by NET blockade (Wang et al., 2015; Nakamura et al., 2019). One plausible explanation for these differences is that each monoamine system possesses distinct release dynamics (e.g., levels of tonic activity, neurotransmitter clearance, etc.). Although addressing these possibilities is beyond the scope of this study, it is worth noting that a NET-specific effect at this synapse in the NAcSh is comparatively less likely in a region densely innervated by SERT-expressing and DAT-expressing terminals. Taken together, we propose a specific synaptic locus within the feedforward microcircuitry that is under selective noradrenergic control by NET.

\section{Noradrenergic modulation of glutamatergic transmission via $\alpha_{2}$-ARs is restricted to PV-IN synapses in the NAcSh}

$\mathrm{NE}$ exerts differential effects on neurotransmission according to $\alpha / \beta$-AR subtype, synaptic locus, and brain region. For example, presynaptic $\alpha_{2}$-AR signaling in the BNST decreases glutamate release probability via canonical heteroreceptor mechanisms, whereas postsynaptic $\alpha_{2 \mathrm{~A}}$-ARs elicit HCN-dependent effects on neuronal output (Shields et al., 2009; Harris et al., 2018). NE signaling in the dorsal striatum potentiates cholinergic IN firing via $\beta_{1}$-ARs through a cAMP-dependent mechanism (Pisani et al., 2003). Our results in the NAcSh indicate that the heterosynaptic actions of NET blockade, or direct NE application, at PV-IN synapses are mediated by postsynaptically-localized $\alpha_{2}$-ARs. This is supported by data showing that the synaptic effects of NE and TMx were unaccompanied by a change in PPR and CV and prevented by GDP disabling agent, $\mathrm{GDP}_{\beta} \mathrm{S}$. Furthermore, the effects of TMx persisted in the presence of a non-selective $\beta$-AR antagonist, were abolished by a selective- $\alpha_{2}$-AR antagonist, and readily recapitulated with an $\alpha_{2 \mathrm{~A}} \mathrm{AR}$ agonist. Interestingly, a higher, supraphysiological concentration of NE $(100 \mu \mathrm{M})$ used to overcome possible removal by NET unmasked an inward current that coincided with a reduction in $\mathrm{R}_{\mathrm{m}}$. Although we do not identify the specific ionic conductances contributing to these membrane parameters, the directionality of $\Delta \mathrm{I}_{\text {Holding, unchanged }}$ $\mathrm{V}_{\mathrm{RMP}}$ and AP fidelity elicited by NE likely points to a postsynaptic ionic mechanism confined to dendritic specializations. It remains unclear whether the identified conductance triggered by $\mathrm{NE}$ at this elevated concentration represents a physiologicallyrelevant modulatory mechanism.

The observation that NE modulates glutamatergic synaptic strength onto $\mathrm{PV}$-INs but not MSNs diverges from prior accounts of NE function in the NAc, with early studies indicating that NE targets $\alpha$-ARs at glutamatergic synapses onto MSNs (Nicola and Malenka, 1998; Peng et al., 2018). Our findings suggest that NE, TMx, and guanfacine had minimal efficacy at glutamatergic synapses onto MSNs. It is worth noting that the aforementioned work was performed in rats with a concentration of NE $10 \times$ that used here, perhaps indicating that synapses onto MSNs become engaged by NE during bouts of sustained noradrenergic activity in the NAcSh. Alternatively, MSNs may undergo NE-dependent plasticity through a polysynaptic mechanism involving other NAc microcircuits. Direct monosynaptic actions at GABAergic synapses are less likely given our findings and others that local eIPSC/Ps are unaffected by NE, highlighting the neuromodulatory importance of upstream synapses in the control of PV-IN-directed FFI. An intriguing proposition is that the limited number of NE-containing varicosities in the NAc corresponds anatomically to the number of PV-INs. Interestingly, studies in vivo indicate the glutamate-induced activation of the NTS leads to increased single-unit activity of NAcSh neurons, with a small subset of single-unit recordings showing decreased activity (Kirouac and Ciriello, 1997). Future studies are needed to delineate the functional consequences of NE signaling at other synaptic units in the NAc, including other IN subtypes and microcircuits.

\section{PFC-evoked feedforward inhibition onto MSNs is discretely regulated by $\alpha_{2}$-ARs}

Functional NAc output is mediated by the coordinated activity of D1 and D2 MSNs. Because MSNs and PV-INs lack intrinsic pacemaker mechanisms, concerted glutamatergic input sustains plateau potentials near AP spike threshold (Kawaguchi, 1993). Therefore, mechanisms that shift excitatory-inhibitory (E/I) balance onto MSNs have profound consequences on downstream limbic-motor units targeted by the NAc. Using optogenetics, we show that PFC afferents to the NAc engage a disynaptic feedforward circuit onto MSNs that is inhibited by CP-AMPAR antagonist, NASPM. Recent studies indicate that the basal expression of CP-AMPARs is restricted to PV-INs (Manz et al., 2020b), indicating that PFC-evoked FFI of MSNs is likely mediated by PVINs, although CP-AMPARs on other GABAergic INs in the NAc cannot be ruled out (Yu et al., 2017). We confirmed the contribution of PV-INs to FFI by reducing glutamatergic drive onto PV-INs using the AMPAR-selective YM90K ${ }^{\text {DART }}$ which largely eliminated PFC-evoked AP firing in PV-INs and attenuated FFI of MSNs. It is critical to note that because (1) YM90K ${ }^{\mathrm{DART}}$ at $300 \mathrm{~nm}$ is subthreshold for complete AMPAR blockade and (2) incomplete penetrance of the YM90K ${ }^{\mathrm{DART}}$ recipient, $\mathrm{HT}$, was observed in our preparation, we did not expect feedforward glutamatergic drive onto PV-INs to be uniformly disabled in the NAcSh. Nevertheless, we saw a robust reduction of FFI in Tom/ $\mathrm{HT}(-)$ MSNs in close proximity to Tom/HT(+) PV-INs.

The PV-IN-specific recruitment of $\alpha_{2}$-ARs prompted us to examine how this mechanism affects the efficacy with which glutamatergic inputs to the NAcSh drive feedforward inhibition. Indeed, $\alpha_{2}$-AR activity decreased PFC-evoked feedforward inhibition onto MSNs without concomitantly altering monosynaptic GABAergic transmission assessed electrically. Furthermore, guanfacine did not consistently modulate FFI when PV-IN AMPARs were blocked by YM90K ${ }^{\mathrm{DART}}$, indicating that $\alpha_{2}$-AR function requires feedforward glutamatergic drive onto PV-INs. The lack of effect of $\alpha_{2}$-AR activity on heterogeneous glutamatergic and GABAergic synapses onto MSNs suggests that upstream plasticity within PV-IN microcircuits is sufficient to bias E/I balance onto MSNs. An unexpected observation from our results is that the magnitude with which $\alpha_{2}$-AR activity decreased PFC-evoked oIPSCs mirrored experiments performed at glutamatergic synapses onto PV-INs. If $\alpha_{2}$-AR activation decreased synaptic efficacy below the synaptically-evoked AP threshold, PFC-evoked FFI of MSNs would be eliminated. While residual PFC-evoked oIPSCs in the presence of guanfacine may reflect mixed signals arising from PV-INs and other INs, an alternative possibility is that changes in synaptic strength onto PVINs results in a proportional, non-binary change in FFI of MSNs. 
These data lend support to the emerging hypothesis that glutamatergic transmission onto PV-INs is a critical computational element regulating NAc circuit output.

These findings set the stage for future studies to determine which behavioral programs recruit $\alpha_{2}$-AR signaling in the $\mathrm{NAcSh}$, and to determine causal roles of this unique motif to behavior. While central NE-containing locus coeruleus projections mediate various stress-related arousal strategies, anatomic studies indicate that the primary source of NE fibers in the NAcSh is the $A_{2} / C_{2}$ cell group in the NTS (Delfs et al., 1998). Accordingly, ascending NTS inputs to various limbic and paralimbic regions, including the NAc, have been implicated in reward-related behavioral tasks (Delfs et al., 2000; Alsene et al., 2010). For example, the reinforcing effects of morphine are diminished in NTS-specific DBH knock-out (KO) mice and are modulated by pharmacological manipulations of NE signaling (Olson et al., 2006). Despite broad behavioral analyses of NE signaling, it remains to be determined how NE signaling leads to synaptic adaptations in circuits subserving motivated behavior. One possibility is that NE transmission in the NAcSh leads to an adaptive desynchronization of locomotor output by gating MSN release from PV-IN-mediated feedforward inhibition. Indeed, PV-IN network activity has been increasingly implicated in constraining goal-directed behavioral strategies (Owen et al., 2018; Pisansky et al., 2019). Clearly, future experiments are needed to discern the full functional relevance of this circuit mechanism in NAc-dependent motivational states.

In conclusion, while the mesolimbic system receives input from NE-containing brainstem nuclei, few studies have examined the synaptic plasticity mechanisms recruited by NE signaling in the NAcSh. We report here that NE selectively modulates glutamatergic synaptic strength within PV-IN-embedded microcircuits to promote MSN escape from feedforward inhibition. By acting through postsynaptically-localized $\alpha_{2}$-ARs, NE decreases glutamatergic transmission onto PV-INs, thereby decreasing the synaptic efficacy with which afferent glutamatergic inputs (e.g., PFC) drive feedforward inhibition onto MSNs. These studies demonstrate a privileged synapse-specific and microcircuit-specific neuromodulatory effect of NE signaling and a potentially druggable target within the NAc for the treatment of pathologic motivational states, including addiction and depression.

\section{References}

Allin R, Russell VA, Lamm MCL, Taljaard JJF (1988) Regional distribution of monoamines in the nucleus accumbens of the rat. Neurochem Res 13:937-942.

Alsene KM, Fallace K, Bakshi VP (2010) Ventral striatal noradrenergic mechanisms contribute to sensorimotor gating deficits induced by amphetamine. Neuropsychopharmacology 35:2346-2356.

Berridge CW, Stratford TL, Foote SL, Kelley AE (1997) Distribution of dopamine beta-hydroxylase-like immunoreactive fibers within the shell subregion of the nucleus accumbens. Synapse 27:230-241.

Beurrier C, Malenka RC (2002) Enhanced inhibition of synaptic transmission by dopamine in the nucleus accumbens during behavioral sensitization to cocaine. J Neurosci 22:5817-5822.

Brog JS, Salyapongse A, Deutch AY, Zahm DS (1993) The patterns of afferent innervation of the core and shell in the "accumbens" part of the rat ventral striatum: immunohistochemical detection of retrogradely transported fluoro-gold. J Comp Neurol 338:255-278.

Delfs JM, Zhu Y, Druhan JP, Aston-Jones GS (1998) Origin of noradrenergic afferents to the shell subregion of the nucleus accumbens: anterograde and retrograde tract-tracing studies in the rat. Brain Res 806:127-140.

Delfs JM, Zhu Y, Druhan JP, Aston-Jones G (2000) Noradrenaline in the ventral forebrain is critical for opiate withdrawal-induced aversion. Nature 403:430-434.
Dobbs LK, Kaplan AR, Lemos JC, Matsui A, Rubinstein M, Alvarez VA (2016) Dopamine regulation of lateral inhibition between striatal neurons gates the stimulant actions of cocaine. Neuron 90:1100-1113.

Fallon JH, Moore RY (1978) Catecholamine innervation of the basal forebrain. IV. Topography of the dopamine projection to the basal forebrain and neostriatum. J Comp Neurol 180:545-580.

Federman AD, Conklin BR, Schrader KA, Reed RR, Bourne HR (1992) Hormonal stimulation of adenylyl cyclase through Gi-protein beta gamma subunits. Nature 356:159-161.

Giustino TF, Fitzgerald PJ, Ressler RL, Maren S (2019) Locus coeruleus toggles reciprocal prefrontal firing to reinstate fear. Proc Natl Acad Sci U S A 116:8570-8575.

Harris NA, Isaac AT, Günther A, Merkel K, Melchior J, Xu M, Eguakun E, Perez R, Nabit BP, Flavin S, Gilsbach R, Shonesy B, Hein L, Abel T, Baumann A, Matthews R, Centanni SW, Winder DG (2018) Dorsal BNST $\alpha 2 \mathrm{~A}$-adrenergic receptors produce $\mathrm{HCN}$-dependent excitatory actions that initiate anxiogenic behaviors. J Neurosci 38:8922-8942.

Heifets BD, Salgado JS, Taylor MD, Hoerbelt P, Cardozo PD, Steinberg EE, Walsh JJ, Sze JY, Malenka RC (2019) Distinct neural mechanisms for the prosocial and rewarding properties of MDMA. Sci Transl Med 11: eaaw6435.

Ingebretson AE, Hearing MC, Huffington ED, Thomas MJ (2018) Endogenous dopamine and endocannabinoid signaling mediate cocaineinduced reversal of AMPAR synaptic potentiation in the nucleus accumbens shell. Neuropharmacology 131:154-165.

Kawaguchi Y (1993) Physiological, morphological, and histochemical characterization of three classes of interneurons in rat neostriatum. J Neurosci 13:4908-4923.

Kerfoot EC, Williams CL (2011) Interactions between brainstem noradrenergic neurons and the nucleus accumbens shell in modulating memory for emotionally arousing events. Learn Mem 18:405-413.

Kirouac GJ, Ciriello J (1997) Medullary inputs to nucleus accumbens neurons. Am J Physiol 273:R2080-R2088.

Kombian SB, Ananthalakshmi KVV, Zidichouski JA, Saleh TM (2009) Substance $P$ and cocaine employ convergent mechanisms to depress excitatory synaptic transmission in the rat nucleus accumbens in vitro. Eur J Neurosci 29:1579-1587.

Lüscher C, Malenka RC (2011) Drug-evoked synaptic plasticity in addiction: from molecular changes to circuit remodeling. Neuron 69:650-663.

Mantsch JR, Baker DA, Funk D, Lê AD, Shaham Y (2016) Stress-induced reinstatement of drug seeking: 20 years of progress. Neuropsychopharmacology 41:335-356.

Manz KM, Baxley AG, Zurawski Z, Hamm HE, Grueter BA (2019) Heterosynaptic GABAB receptor function within feedforward microcircuits gates glutamatergic transmission in the nucleus accumbens core. J Neurosci 39:9277-9293.

Manz KM, Becker JC, Grueter CA, Grueter BA (2020a) Histamine H3 receptor function biases excitatory gain in the nucleus accumbens. Biol Psychiatry 89:588-599.

Manz KM, Ghose D, Turner BD, Taylor A, Becker J, Grueter CA, Grueter BA (2020b) Calcium-permeable AMPA receptors promote endocannabinoid signaling at parvalbumin interneuron synapses in the nucleus accumbens core. Cell Rep 32:107971.

Mathur BN, Capik NA, Alvarez VA, Lovinger DM (2011) Serotonin induces long-term depression at corticostriatal synapses. J Neurosci 31:74027411.

Matsui A, Alvarez VA (2018) Cocaine inhibition of synaptic transmission in the ventral pallidum is pathway-specific and mediated by serotonin. Cell Rep 23:3852-3863.

McKittrick CR, Abercrombie ED (2007) Catecholamine mapping within nucleus accumbens: differences in basal and amphetamine-stimulated efflux of norepinephrine and dopamine in shell and core. J Neurochem 100:1247-1256.

Mitrano DA, Schroeder JP, Smith Y, Cortright JJ, Bubula N, Vezina P, Weinshenker D (2012) $\alpha$-1 Adrenergic receptors are localized on presynaptic elements in the nucleus accumbens and regulate mesolimbic dopamine transmission. Neuropsychopharmacology 37:2161-2172.

Mitrano DA, Jackson K, Finley S, Seeley A (2018) $\alpha 1$ b-adrenergic receptor localization and relationship to the D1-dopamine receptor in the rat nucleus accumbens. Neuroscience 371:126-137.

Nakamura Y, Dryanovski DI, Kimura Y, Jackson SN, Woods AS, Yasui Y, Tsai S-Y, Patel S, Covey DP, Su TP, Lupica C (2019) Cocaine-induced 
endocannabinoid signaling mediated by sigma-1 receptors and extracellular vesicle secretion. Elife 8:e47209.

Nardou R, Lewis EM, Rothhaas R, Xu R, Yang A, Boyden E, Dölen G (2019) Oxytocin-dependent reopening of a social reward learning critical period with MDMA. Nature 569:116-120.

Nicola SM, Malenka RC (1998) Modulation of synaptic transmission by dopamine and norepinephrine in ventral but not dorsal striatum. J Neurophysiol 79:1768-1776.

Nicola SM, Kombian SB, Malenka RC (1996) Psychostimulants depress excitatory synaptic transmission in the nucleus accumbens via presynaptic D1-like dopamine receptors. J Neurosci 16:1591-1604.

Olson VG, Heusner CL, Bland RJ, During MJ, Weinshenker D, Palmiter RD (2006) Role of noradrenergic signaling by the nucleus tractus solitarius in mediating opiate reward. Science 311:1017-1020.

Owen SF, Berke JD, Kreitzer AC (2018) Fast-spiking interneurons supply feedforward control of bursting, calcium, and plasticity for efficient learning. Cell 172:683-695.e15.

Park JW, Bhimani RV, Park J (2017) Noradrenergic Modulation of Dopamine Transmission Evoked by Electrical Stimulation of the Locus Coeruleus in the Rat Brain. ACS Chem Neurosci 8:1913-1924.

Peng SY, Li B, Xi K, Wang JJ, Zhu JN (2018) Presynaptic $\alpha 2$-adrenoceptor modulates glutamatergic synaptic transmission in rat nucleus accumbens in vitro. Neurosci Lett 665:117-122.

Pisani A, Bonsi P, Centonze D, Martorana A, Fusco F, Sancesario G, De Persis C, Bernardi G, Calabresi P (2003) Activation of beta1-adrenoceptors excites striatal cholinergic interneurons through a cAMP-dependent, protein kinase-independent pathway. J Neurosci 23:5272-5282.

Pisansky MT, Lefevre EM, Retzlaff CL, Trieu BH, Leipold DW, Rothwell PE (2019) Nucleus accumbens fast-spiking interneurons constrain impulsive action. Biol Psychiatry 86:836-847.

Sánchez-Soto M, Bonifazi A, Cai NS, Ellenberger MP, Newman AH, Ferré S, Yano H (2016) Evidence for noncanonical neurotransmitter activation: norepinephrine as a dopamine D2-like receptor agonist. Mol Pharmacol 89:457-466.

Sánchez-Soto M, Casadó-Anguera V, Yano H, Bender BJ, Cai N-S, Moreno E, Canela EI, Cortés A, Meiler J, Casadó V, Ferré S (2018) $\alpha 2 \mathrm{~A}$ - and $\alpha 2 \mathrm{C}$-adrenoceptors as potential targets for dopamine and dopamine receptor ligands. Mol Neurobiol 55:8438-8454.

Scudder SL, Baimel C, Macdonald EE, Carter AG (2018) Hippocampalevoked feedforward inhibition in the nucleus accumbens. J Neurosci 38:9091-9104.

Shields AD, Wang Q, Winder DG (2009) alpha2A-adrenergic receptors heterosynaptically regulate glutamatergic transmission in the bed nucleus of the stria terminalis. Neuroscience 163:339-351.

Shields BC, Kahuno E, Kim C, Apostolides PF, Brown J, Lindo S, Mensh BD, Dudman JT, Lavis LD, Tadross MR (2017) Deconstructing behavioral neuropharmacology with cellular specificity. Science 356:eaaj2161.

Turner BD, Rook JM, Lindsley CW, Conn PJ, Grueter BA (2018) mGlu1 and mGlu5 modulate distinct excitatory inputs to the nucleus accumbens shell. Neuropsychopharmacology 43:2075-2082.

Wang H, Treadway T, Covey DP, Cheer JF, Lupica CR (2015) Cocaineinduced endocannabinoid mobilization in the ventral tegmental area. Cell Rep 12:1997-2008.

Wang X, Gallegos DA, Pogorelov VM, O'Hare JK, Calakos N, Wetsel WC, West AE (2018) Parvalbumin interneurons of the mouse nucleus accumbens are required for amphetamine-induced locomotor sensitization and conditioned place preference. Neuropsychopharmacology 43:953-963.

Wright WJ, Schlüter OM, Dong Y (2017) A feedforward inhibitory circuit mediated by CB1-expressing fast-spiking interneurons in the nucleus accumbens. Neuropsychopharmacology 42:1146-1156.

Yu J, Yan Y, Li K-L, Wang Y, Huang YH, Urban NN, Nestler EJ, Schlüter OM, Dong Y (2017) Nucleus accumbens feedforward inhibition circuit promotes cocaine self-administration. Proc Natl Acad Sci USA 114: E8750-E8759. 